\title{
THE CONORMAL DERIVATIVE PROBLEM FOR EQUATIONS OF VARIATIONAL TYPE IN NONSMOOTH DOMAINS
}

\author{
GARY M. LIEBERMAN
}

\begin{abstract}
It is well known that elliptic boundary value problems in smooth domains have smooth solutions, but if the domain is, say, $C^{1}$, the solutions need not be Lipschitz. Recently Korevaar has identified a class of Lipschitz domains, in which solutions of the capillary problem are Lipschitz assuming the contact angle relates correctly to the geometry of the domain. Lipschitz bounds for more general boundary value problems in the same class of domains are proved. Applications to variational inequalities are also considered.
\end{abstract}

According to the standard theory $[5,10]$, solutions of elliptic boundary problems in $C^{k, \alpha}$ domains $(k \geq 2)$ are also $C^{k, \alpha}$ up to the boundary of the domain; more recent work $[2,4,11,16,17,19]$ has shown that this statement is true for $k=1$ also. However, if the domain is $C^{1}$, even if all other data of the problem are $C^{\infty}$, the solution need not even be Lipschitz (see [8]). As can be seen for harmonic functions in a sector, for Lipschitz domains the connection between smoothness of the solution and that of the domain is more delicate. Here we investigate this connection for a family of boundary value problems modelled on the capillary problem in a domain with corners. We prove gradient bounds for this family of problems via a combination of Korevaar's approach to the capillary problem in nonsmooth domains $[9, \S 4]$ and the author's gradient estimates in smooth domains $[12,21]$ which, in turn, are based on the interior estimates of Simon [28] and the global estimates of Ural'tseva for the capillary problem [29]. The important point is to verify that the considerations of those works apply to our situation.

Specifically, for $\Omega$ a bounded domain in $\mathbb{R}^{n}$ with unit inner normal $\gamma$ and appropriate functions $A^{1}, \ldots, A^{n}, B: \Omega \times \mathbb{R} \times \mathbb{R}^{n} \rightarrow \mathbb{R}$, and $\varphi: \partial \Omega \times \mathbb{R} \rightarrow \mathbb{R}$, we consider the problem

$$
\begin{aligned}
\operatorname{div} A(x, u, D u)+B(x, u, D u) & =0 & & \text { in } \Omega, \\
A(x, u, D u) \cdot \gamma+\varphi(x, u) & =0 & & \text { on } \partial \Omega .
\end{aligned}
$$

Our primary hypotheses are: $\Omega$ is Lipschitz and satisfies a uniform exterior sphere condition, $\partial \Omega$ is $C^{2}$ except for a small set, and $A(x, z, p)=$ $\partial F(x, z, p) / \partial p$ for a scalar function $F$ which is convex in $p$. The conditions on $\Omega$ come from [9] while the existence of $F$ is the definition of variational

Received by the editors October 27, 1989.

1980 Mathematics Subject Classification (1985 Revision). Primary 35J65; Secondary 49A29.

Key words and phrases. Quasilinear elliptic equations, conormal derivative, boundary value problems, variational inequalities. 
type. The latter hypothesis was used in [12] and then dispensed with in [21] for smooth domains. Its present relevance will be discussed later. Of course the convexity of $F$ guarantees that the matrix $\left(\frac{\partial A^{i}}{\partial p_{j}}\right)$ is positive definite and hence the boundary value problem is elliptic.

Various technical hypotheses will be made on the structure of the functions $F, A, B, \varphi$, but we note here one particular such hypothesis for the capillary problem in two dimensions. In this case $F=\left(1+|p|^{2}\right)^{1 / 2}$ and $A=$ $\left(1+|p|^{2}\right)^{-1 / 2} p$, so $|A|<1$. Therefore a necessary condition for $(0.1)$ to have smooth solutions is that $|\varphi|<1$. If also $\varphi$ is Lipschitz with $\varphi_{z} \leq 0$, it follows from [21, Exampie 1, p. 57] that $|D u|$ is bounded for domains with smooth boundaries. (When $\varphi_{z}=0$, this result is due to Gerhardt [3] and Ural'tseva [29].) At a convex corner, even for constant $\varphi$, the condition $|\varphi|<1$ is not strong enough to give a bound on $|D u|$ or, in fact, $|u|$. (Details of this situation are given in, e.g., $[1, \S 5.2 ; 9$, p. 20].) One crucial condition which gives these bounds is the existence of smooth functions $\bar{\varphi}$ (scalar) and $\bar{\gamma}$ (vector) with $|\bar{\varphi}|<1,|\bar{\gamma}| \leq 1$, and $\varphi=\bar{\gamma} \cdot \gamma \bar{\varphi}$. Korevaar introduced this condition in [9], and he called $\bar{\gamma}$ a pseudonormal, a term we also use here.

An interesting comparison can be made between the results here and those in other works of the author. Suppose $\Omega$ is a cube in $\mathbb{R}^{3}, \varphi=0, A^{i}(x, z, p)=$ $a^{i j} p_{j}$ for some constant positive-definite matrix $\left(a^{i j}\right)$, and $B$ is $C^{\infty}(\bar{\Omega})$ and depends only on $x$. The results of [20] or [22] give a constant $\alpha \in(0,1)$ determined only by $\left(a^{i j}\right)$ such that solutions of $(0.1)$ are $C^{1, \alpha}$ uniformly on any compact subset of $\bar{\Omega}$ minus its vertices. Global Hölder continuity of the solution is classical, and results in this paper will show global Lipschitz continuity if $\left(a^{i j}\right)$ is symmetric.

Unfortunately some symmetry is crucial to the present approach. As in all gradient estimates, the key step is to find a function of the gradient which is a subsolution of a suitable boundary value problem. (For example if $u$ is harmonic, then $|D u|^{2}$ is subharmonic; see [27] for further discussion in case a boundary gradient estimate is known.) In our case we consider a function $v_{0}(x, u, D u)$ which is convex and of quadratic growth in $D u$. (For technical reasons, it will be convenient to use linear rather than quadratic growth in the main body of this paper, but the quadratic growth is useful for illustration.) In addition, $v_{0}$ must have a suitable form to take advantage of the structure of the differential equation and the boundary condition. Among other things we would like the vector field $\frac{\partial v_{0}}{\partial p}$ to be tangential to the boundary. Then, writing $a^{i j}$ for $\frac{\partial A^{i}}{\partial p_{j}}$ and differentiating the boundary condition in the tangential direction $\frac{\partial v_{0}}{\partial p}$ yields

or

$$
a^{i j} \gamma_{i} D_{j k} u \frac{\partial v_{0}}{\partial p_{k}}=\text { lower order terms }
$$

$$
a^{i j} \gamma_{i} D_{j} v_{0}=\text { lower order terms }
$$

on $\partial \Omega$ and then the differential equation gives

$$
D_{i}\left(a^{i j} D_{j} v_{0}\right)=\text { lower order terms }
$$

in $\Omega$. Therefore $v_{0}$ satisfies a conormal derivative problem, which leads to estimates on $v_{0}$ (and hence on $|D u|$ ) after appropriate estimation of the lower order terms. 
To return to the role of symmetry, we suppose now for simplicity that $A^{i}(x, z, p)=a^{i j} p_{j}$ for some constant positive-definite matrix $a^{i j}$, with $a^{21}=$ 0 , that $\varphi \equiv 0$, and that $\Omega$ is two dimensional. Also, suppose first also that $\partial \Omega$ is the hyperplane $\left\{x^{2}=0\right\}$. As noted in [15], we can take

$$
v_{0}=p_{1}^{2}+p_{2}^{2}
$$

since $\frac{\partial v_{0}}{\partial p_{2}}=0$ on $\partial \Omega$. Suppose next that $\partial \Omega$ is the angle $\left\{x^{2}=0, x^{1} \geq\right.$ $0\} \cup\left\{x^{1}=0, x^{2}>0\right\}$. If $v_{0}=b^{i j} p_{i} p_{j}$ for some constant positive-definite $\left(b^{i j}\right)$, which we may assume symmetric, the boundary condition on $\left\{x^{2}=0\right\}$ and $\frac{\partial v_{0}}{\partial p}$ being tangential give

$$
p_{2}=0 \text { implies } b^{21} p_{1}+b^{22} p_{2}=0
$$

and hence $b^{21}=0$ since $p_{1}$ is arbitrary. Thus on $\left\{x^{1}=0\right\}$ we have

$$
a^{11} p_{1}+a^{12} p_{2}=0 \text { implies } b^{11} p_{1}=0,
$$

which is only possible if $a^{12}=0$, i.e., $\left(a^{i j}\right)$ is symmetric. Such a hypothesis is not needed in the case of a domain with smooth boundary. Thus we see that the present approach is not easily modified to remove the symmetry hypothesis on $a^{i j}$ (or, equivalently, the variational hypothesis on $A$ ).

We begin in $\S 1$ with some basic inequalities which are used to prove estimates on solutions of $(0.1)$ in $\S 2$ and on their gradients in $\S 3$. Analogous results for parabolic problems are stated in $\S 4$. Examples in $\S 5$ illustrate our structure conditions and the role of the pseudonormal, and connections with variational inequalities appear in $\S 6$. Finally the progression from our estimates to regularity results is made, via existence theorems, in $\S 7$.

In general our notation follows [5, 12]. Unless otherwise indicated the arguments of $A, B$, and their derivatives are taken to be $(x, u, D u)$ and hence omitted. Similarly $\varphi=\varphi(x, u)$. The subscripts $x, z, p$ denote derivatives with respect to the appropriate scalar or vector variables. Other subscripts denote derivatives with respect to components of $x$ or components of a vector or tensor, and superscripts denote derivatives with respect to components of $p$ or components of a vector or tensor. The meaning should be clear from the context, e.g., $A_{k}^{i}=\frac{\partial A^{i}}{\partial x^{k}}, B^{j}=\frac{\partial B}{\partial p_{j}}$.

\section{Preliminaries}

We begin by proving two inequalities which relate certain boundary and interior integrals. These inequalities are analogs of the standard ones proved as Lemma 2.1 in [12], but additional complications arise when investigating nonsmooth domains. A complete understanding of these complications is intimately tied to several of our structure conditions, so the results in this section will not be as independent of the gradient estimates as those in [12, §2].

Throughout we assume $\Omega$ to be a Lipschitz domain and $\bar{\gamma}$ to be a $C^{0,1}(\bar{\Omega})$ vector field with $|\bar{\gamma}| \leq 1$ in $\Omega$ and $\bar{\gamma} \cdot \gamma \geq 0$ almost everywhere with respect to surface measure $d s$ on $\partial \Omega$.

Lemma 1.1. If $K=\sup _{\Omega}(\operatorname{div} \bar{\gamma})_{-}$, then, for all nonnegative $h \in W^{1,1}$,

$$
\int_{\partial \Omega} h \gamma \cdot \bar{\gamma} d s \leq \int_{\Omega}|D h| d x+K \int_{\Omega} h d x .
$$


Proof.

$$
\int_{\partial \Omega} h \gamma \cdot \bar{\gamma} d s=-\int_{\Omega} \operatorname{div}(h \bar{\gamma}) d x=-\int_{\Omega} D h \cdot \bar{\gamma} d x-\int_{\Omega} h \operatorname{div} \bar{\gamma} d x .
$$

Note that such a $\bar{\gamma}$ can be constructed for an arbitrary $C^{0,1}$ domain very simply. Locally $\partial \Omega$ is the graph of a Lipschitz function, so we can choose $\bar{\gamma}$ locally to be constant (in the obvious direction) and then patch these together via a smooth partition of unity.

Our next lemma involves the boundary condition from $(0.1)$ and some additional notation. For $u \in W^{2,1} \cap C^{0,1}(\bar{\Omega})$ and $h \in W^{1,1}$ we define

$$
\begin{gathered}
v=\sqrt{1+|D u|^{2}}, \quad \nu=D u / v, \quad g^{i j}=\delta^{i j}=\delta^{i j}-\nu_{i} \nu_{j}, \\
\delta_{i} h=g^{i j} D_{j} h, \quad d \mu=v d x
\end{gathered}
$$

and for a $C^{0,1}$ vector function $A$ defined on $\bar{\Omega} \times \mathbb{R} \times \mathbb{R}^{n}$, we define

$$
a^{i j}=v \frac{\partial A^{i}}{\partial p_{j}}(x, u, D u)
$$

Lemma 1.2. Suppose

$$
A(x, u, D u) \cdot \gamma+\varphi(x, u)=0 \text { on } \partial \Omega
$$

and there is a function $\bar{\varphi}$ such that

$$
|\bar{\varphi}||D u| \leq \beta_{1} D u \cdot A(x, u, D u) \quad \text { and } \quad \varphi=\bar{\varphi} \gamma \cdot \bar{\gamma} \quad \text { on } \partial \Omega
$$

for some constant $\beta_{1} \in[0,1)$. If $K$ is a constant such that

$$
K \geq|D \bar{\gamma}| \quad \text { in } \Omega,
$$

then, for any nonnegative $h \in W^{1,1}$, we have

$$
\begin{aligned}
& \int_{\partial \Omega} h \bar{\gamma} \cdot \gamma D u \cdot \frac{A}{|A|} d s \\
& \leq C\left(\beta_{1}\right)\left[\int_{\Omega}(|\delta h|+K h) d \mu\right. \\
& \left.\quad+\int_{\Omega} h\left(\nu \cdot \bar{\gamma} D_{i}\left(\frac{A^{i}}{|A|}\right)-\bar{\gamma}^{i} \nu_{j} D_{i}\left(\frac{A^{j}}{|A|}\right)\right) d \mu\right] .
\end{aligned}
$$

Proof. Set

$$
I=\int_{\partial \Omega} h\left[D u \cdot \frac{A}{|A|} \bar{\gamma}-D u \cdot \bar{\gamma} \frac{A}{|A|}\right] \cdot \gamma d s .
$$

(This integral, in disguised form, was used in the proof of [30, Lemma 2].) The inequality in (1.5) gives

$$
I \geq\left(1-\beta_{1}\right) \int_{\partial \Omega} h \bar{\gamma} \cdot \gamma D u \cdot \frac{A}{|A|} d s .
$$


The divergence theorem implies that

$$
\begin{aligned}
I= & -\int_{\Omega}\left(D u \cdot \frac{A}{|A|} \bar{\gamma}-D u \cdot \bar{\gamma} \frac{A}{|A|}\right) \cdot D h d x \\
& -\int_{\Omega} h\left(\frac{A^{j}}{|A|} D_{i j} u \bar{\gamma}^{i}-\bar{\gamma}^{j} D_{i j} u \frac{A^{i}}{|A|}\right) d x \\
& -\int_{\Omega} h\left(D u \cdot \frac{A}{|A|} \operatorname{div} \bar{\gamma}-\frac{A^{i}}{|A|} D_{i} \bar{\gamma}^{j} D_{j} u\right) d x \\
& -\int_{\Omega} h\left(\bar{\gamma}^{i} D_{j} u D_{i}\left(\frac{A^{j}}{|A|}\right)-\bar{\gamma} \cdot D u D_{i}\left(\frac{A^{i}}{|A|}\right)\right) d x \\
= & -\int_{\Omega}\left(\nu \cdot \frac{A}{|A|} \bar{\gamma}-\nu \cdot \bar{\gamma} \frac{A}{|A|}\right) \cdot \delta h d \mu \\
& -\int_{\Omega} h\left(\nu \cdot \frac{A}{|A|} \operatorname{div} \bar{\gamma}-\frac{A^{i}}{|A|} D_{i} \bar{\gamma}^{j} \nu_{j}\right) d \mu \\
& +\int_{\Omega} h\left(\nu \cdot \bar{\gamma} D_{i}\left(\frac{A^{i}}{|A|}\right)-\bar{\gamma}^{i} \nu_{j} D_{i}\left(\frac{A^{j}}{|A|}\right)\right) d \mu
\end{aligned}
$$

because $D u \cdot A \bar{\gamma} \cdot \nu=D u \cdot \bar{\gamma} A \cdot \nu$ and $A^{j} D_{i j} u \bar{\gamma}^{i}=\bar{\gamma}^{j} D_{i j} u A^{i}$. Now invoking (1.6) and (1.8) yields (1.7).

From the proof it is clear that the inequality in (1.5) need only hold at points of $\partial \Omega$ for which $h>0$.

\section{BOUNDS ON THE SOLUTION}

In this section, we give pointwise estimates for solutions of $(0.1)$. Since the proofs are the same as for the corresponding results in [12, §3] (see also [21, $\S 2])$ with Lemma 1.1 replacing [12, Lemma $2.1(\mathrm{a})]$, we only state the results.

Lemma 2.1. Let $u \in C^{0,1}$ be a weak solution of (0.1). Suppose there are positive constants $m>1, a_{1}, b_{0}, b_{1}, c_{0}, M$ such that

$$
\begin{gathered}
p \cdot A(x, z, p) \geq|p|^{m}-a_{1}|z|^{m}, \\
z B(x, z, p) \leq b_{0} p \cdot A(x, z, p)+b_{1}|z|^{m}, \\
z \varphi(x, z) \leq c_{0}|z|^{m} \quad \text { for } x \in \partial \Omega
\end{gathered}
$$

for $|z| \geq M$. Then, for any $q \geq 1$,

$$
\begin{aligned}
\sup |u| \leq & C(m, n, q, \Omega) \\
& \times\left(\left[\left(1+a_{1}+b_{1}+c_{0}^{m /(m-1)}\right)^{n / m} \int_{\Omega}|u|^{q} d x\right]^{1 / q}+M\right) .
\end{aligned}
$$

Lemma 2.2. Let $u \in C^{0,1}$ be a weak solution of $(0.1)$ and suppose there are positive constants $m>1, m_{0} \leq m, a_{1}, b_{0}, b_{1}, c_{0}, M_{0}$ such that

$$
\begin{gathered}
p \cdot A(x, z, p) \geq|p|^{m}-a_{1}|z|^{m_{0}}, \\
z B(x, z, p) \leq b_{0} p \cdot A(x, z, p)-b_{1}|z|^{m_{0}},
\end{gathered}
$$




$$
z \varphi(x, z) \leq c_{0}|z|^{m_{0}} \quad \text { for } x \in \partial \Omega .
$$

If $q \geq 1$ and if $b_{1}$ is sufficiently large (depending only on $a_{1}, b_{0}, c_{0}, q, m, \Omega$ ), then

$$
\int_{\Omega}|u|^{q} d x \leq 4^{q / m_{0}} M_{0}^{q}|\Omega| .
$$

We emphasize that Lemmata 2.1 and 2.2 are valid for arbitrary Lipschitz domains. For the capillary problem, a bound on $z \varphi$ is not enough to guarantee a bound on $u$ unless the bound on $z \varphi$ is tied into the geometry of $\Omega$. The pseudonormal $\bar{\gamma}$ gives a natural connection.

Lemma 2.3. Let $u \in C^{0,1}$ be a weak solution of $(0.1)$ and suppose there are positive constants $a_{1}, b_{0}, b_{1}, M$ such that $(2.1 \mathrm{a}, \mathrm{b})$ hold with $m=1$ and $|z|>$ $M$. If there is $c_{0} \in(0,1)$ such that

$$
z \phi(x, z) \leq c_{0} \bar{\gamma} \cdot \gamma|z| \text { for } x \in \partial \Omega,|z| \geq M,
$$

then, for any $q \geq 1$

$$
\sup |u| \leq C\left(c_{0}, n, q, \Omega\right)\left[\left(\left(1+a_{1}+b_{1}\right)^{n} \int_{\Omega}|u|^{q} d x\right)^{1 / q}+M\right] .
$$

Lemma 2.4. Lemma 2.2 remains valid for $m=1$ if $(2.1 \mathrm{c})^{\prime \prime}$ replaces $(2.1 \mathrm{c})^{\prime}$ and $c_{0}<1$.

\section{THE GRADIENT BOUND}

We are now ready to derive gradient bounds for solutions of $(0.1)$. In addition to the notation of $\S 1$, we use $\beta, c, \gamma_{0}, K, M, \varphi_{0}$ to denote nonnegative constants with

$$
|u| \leq M \quad \text { and }|\bar{\varphi}(x, u)| \leq \varphi_{0} \quad \text { in } \Omega, \quad \gamma \cdot \bar{\gamma} \geq \gamma_{0}>0 \quad \text { on } \partial \Omega
$$

(recall $\varphi=\overline{\varphi \gamma} \cdot \gamma$ on $\partial \Omega$ ); $\beta, c, K$ will often be subscripted as well.

We assume that there is a $C^{1}$ increasing function $v_{1}$ such that

$$
0<\beta_{0} v \leq v_{1}(F(x, u, D u)+\bar{\varphi} D u \cdot \bar{\gamma}) \leq v
$$

in $\Omega$ and we also write $v_{1}$ for $v_{1}(F+\bar{\varphi} D u \cdot \bar{\gamma})$.

We define

$$
\mathscr{C}^{2}=\frac{1}{v^{2}} a^{i j} a^{k m} D_{j k} u D_{i m} u, \quad \mathscr{E}=\frac{1}{v^{2} v_{1}^{\prime}} a^{i j} D_{i} v_{1} D_{j} v_{1},
$$

and for $\tau \geq \beta_{0}, x_{0} \in \Omega$, and $\rho>0$,

$$
\begin{gathered}
\Omega_{\tau}=\left\{x \in \Omega: v_{1}>\tau\right\}, \quad \Gamma_{\tau}=\left\{x \in \partial \Omega: v_{1}>\tau\right\}, \\
B_{\rho}=\left\{x \in \mathbb{R}^{n}:\left|x-x_{0}\right|<\rho\right\}, \quad \Omega_{\tau, \rho}=B_{\rho} \cap \Omega_{\tau}, \quad \Gamma_{\tau, \rho}=B_{\rho} \cap \Gamma_{\tau} .
\end{gathered}
$$

Next we introduce structure functions $w, \lambda, \lambda_{0}, \Lambda, \Lambda_{0}, \Lambda_{1}$, which are positive, increasing, and (except for $\Lambda_{0}$ ) $C^{1}$ on $\left[\tau_{0}, \infty\right)$ for some $\tau_{0} \geq \beta_{0}$. These functions are related by the monotonicity conditions

$$
w^{\beta}(\Lambda / \lambda)^{(n+2) / 2} / \Lambda \text { is increasing, }
$$

$$
w^{\beta} / \lambda_{0} \text { is increasing, }
$$




$$
\xi^{-\beta}(\Lambda / \lambda)^{(n+2) / 2} / \Lambda, \xi^{-\beta} w \text { are decreasing }
$$

(for $\xi$ the identity function), and

$$
\begin{gathered}
\lambda_{0}^{2} \leq \Lambda_{0} \leq \Lambda, \\
\lambda_{0} \leq \Lambda_{1} \\
\left(\xi \Lambda_{1}^{\prime}\right)^{2}+\Lambda_{1}^{2} \leq \Lambda_{0} .
\end{gathered}
$$

All of our conditions are assumed to hold on $\Omega_{\tau_{0}}$ (or $\Gamma_{\tau_{0}}$ for $(3.8 \mathrm{~d}, \mathrm{f})$ ). First, we assume $\left(a^{i j}\right)$ to be elliptic on $\Omega_{\tau_{0}}$, so there are positive functions $\bar{\mu} \geq \underline{\mu}$ such that

$$
\underline{\mu}|\zeta|^{2} \leq a^{i j} \zeta_{i} \zeta_{j} \leq \bar{\mu}|\zeta|^{2} \quad \text { for all } \zeta \in \mathbb{R}^{n} .
$$

Moreover, we assume that

$$
\begin{gathered}
\lambda_{0} g^{i j} \zeta_{i} \zeta_{j} \leq a^{i j} \zeta_{i} \zeta_{j}, \\
\lambda\left(1+\left(v_{1} \lambda^{\prime} / \lambda\right)^{2}\right) g^{i j} \zeta_{i} \zeta_{j} \leq \lambda_{0} a^{i j} \zeta_{i} \zeta_{j}
\end{gathered}
$$

for $\zeta \in \mathbb{R}^{n}$, and that

$$
\lambda_{0} \leq|A+\overline{\varphi \gamma}| \leq \beta_{1} \lambda_{0}
$$

$$
\lambda_{0} \bar{\mu} \leq \Lambda_{0},
$$

$$
\lambda \bar{\mu} \leq \lambda_{0}^{3}, \quad \lambda \leq \lambda_{0} \bar{\mu} .
$$

Furthermore, we assume that

$$
D u \cdot A \geq \beta_{2} \varphi_{0} v, \quad D u \cdot A \geq \beta_{3}|A| v
$$

with $\beta_{2}>1$ and $\beta_{3}>0$.

Next, we suppose that there are functions $C_{k}^{i}$ and $D_{k}^{i}$ with $D_{k}^{i}$ differentiable with respect to $(x, z, p)$ such that

$$
D_{k} u A_{z}^{i}+A_{k}^{i}+B \delta_{k}^{i}=C_{k}^{i}+D_{k}^{i},
$$

(here $D_{k}^{i j}=\partial D_{k}^{i} / \partial p_{j}$ and $\left.D_{k i}^{i}=\partial D_{k}^{i} / \partial x^{i}\right)$,

$$
\begin{gathered}
\sum_{i, k}\left|C_{k}^{i}\right|^{2} \leq \beta_{4}^{2} \lambda_{0} \Lambda_{0} / \bar{\mu}, \quad \sum_{k}\left|C_{k}^{i} \nu_{i}\right|^{2} \leq \beta_{4}^{2} \underline{\mu} \Lambda_{0} / \lambda_{0} \\
v^{2} \sum_{i, j}\left|D_{k}^{i j}\left(A^{k}+\overline{\varphi \gamma}^{k}\right)\right|^{2} \leq \beta_{4}^{2} \Lambda_{0} \\
\sum_{i}\left|D_{k}^{i j} D_{j} u\left(A^{k}+\overline{\varphi \gamma}^{k}\right)\right|^{2} \leq \beta_{4}^{2} \underline{\mu} \Lambda_{0} / \lambda_{0}
\end{gathered}
$$

$$
\left(D_{i} u D_{k z}^{i}+D_{k i}^{i}\right)\left(A^{k}+\overline{\varphi \gamma}^{k}\right) \leq \beta_{4}^{2} \Lambda_{0}
$$

$$
-D_{k}^{i} \gamma_{i}\left(A^{k}+\overline{\varphi \gamma}^{k}\right) \leq \beta_{4} \lambda_{0} \Lambda_{1},
$$




$$
\begin{gathered}
\left|\bar{\varphi}_{x}\right| \leq \beta_{4} \lambda_{0}, \quad|D \bar{\gamma}| \leq \beta_{4}, \\
\bar{\varphi}_{z}(D u \cdot A+\bar{\varphi} D u \cdot \bar{\gamma}) \leq \beta_{4} \lambda_{0} \Lambda_{1}, \\
v^{2}\left|A_{x}\right|^{2}+\left|F_{x}\right|^{2} \leq \beta_{4}^{2} v^{2} \lambda_{0} \Lambda_{0} / \bar{\mu}, \\
a^{i j} D_{i} u D_{j} u\left(\left|A_{z}\right|^{2}+\left|\bar{\varphi}_{z}\right|^{2}+v^{-2} F_{z}^{2}\right) \leq \beta_{4}^{2} \lambda_{0} \Lambda_{0}, \\
(\Lambda / \lambda)^{(n+2) / 2} v \leq \beta_{5} w^{\beta_{6}} D u \cdot A, \\
v_{1}\left(w^{\prime}\right)^{2} \lambda_{0}^{2} \leq \beta_{7}^{2} \underline{\mu} D u \cdot A, \\
\lambda_{0} w \leq \beta_{7} D u \cdot A, \\
\bar{\mu} v \leq \beta_{7}^{2} D u \cdot A, \\
\Lambda_{0} v_{1} \leq \varepsilon\left(v_{1}\right) w^{2} \lambda_{0} D u \cdot A, \\
v\left|A_{z}\right|+\left|A_{x}\right| \leq \beta_{8} \varepsilon\left(v_{1}\right)^{1 / 2} D u \cdot A
\end{gathered}
$$

for some positive decreasing function $\varepsilon$, and

$$
B \geq-\beta_{9} D u \cdot A,
$$

$$
|A| \leq \varepsilon_{1}(v) D u \cdot A \text { for } v \geq \tau .
$$

Let us point out that these conditions are essentially the same as in [12 and 21] (except that the latter work does not use the variational structure). In particular, our $(3.10 \mathrm{a})$ is the same as [21, (3.12a)] (in which the exponent 2 for $\left(w^{\prime}\right)$ is missing) by virtue of $[12,(4.11 \mathrm{~b})]$ and $(3.1)$.

When $\partial \Omega$ is not smooth, we need some additional control of its geometry beyond being Lipschitz. Following [9], we assume also that it satisfies a uniform exterior sphere condition with radius $R$ such that

$$
R \beta_{4} \geq 1,
$$

and that $\partial \Omega$ is $C^{2}$ except for a singular set $\Gamma$ of finite $(n-2)$-dimensional Hausdorff measure. In particular, there is a sequence of $C^{1}$ functions $\left(\theta_{k}\right)$ such that

$$
\theta_{k}=0 \quad \text { on } \Gamma, 0 \leq \theta_{k} \leq 1, \quad\left\|D \theta_{k}\right\|_{L^{2}} \leq K_{0}(\Gamma) \text { for all } k,
$$

and $\theta_{k} \rightarrow 1$ a.e. For $n>2$, the existence of such a sequence follows from [24, Theorem 21 and 26, Lemma 8]. For $n=2$, the sequence can be constructed easily because $\Gamma$ consists of finitely many points. Furthermore, as we prove in the appendix, (3.12) implies

$$
D_{i} \gamma_{j} \zeta^{i} \zeta^{j} \leq|\zeta|^{2} / R \quad \text { on } \partial \Omega \backslash \Gamma
$$

for any vector $\zeta$ with $\zeta \cdot \gamma=0$. In fact, we shall see in $\S 7$ that this condition, and not the size of the singular set, is what we use. 
Lemma 3.1. Let $\chi$ be a nonnegative Lipschitz function on $\left[\tau_{0}, \infty\right)$, let $\tau \geq \tau_{0}$ and suppose there is a constant $c_{\chi}$ such that

$$
0 \leq(t-\tau) \chi^{\prime}(t) / \chi(t) \leq c_{\chi} \text { for } t \geq \tau
$$

If $\zeta \in C^{0,1}(\bar{\Omega})$ and if conditions (3.3a, c), (3.4), (3.5a, c, d, e), (3.6), (3.7), and (3.8) hold, then

$$
\begin{aligned}
& \int_{\Omega_{\tau}} {\left[\left(1-\frac{\tau}{v_{1}}\right) \mathscr{C}^{2}+\mathscr{E}\right] \chi \zeta^{2} d \mu } \\
& \quad \leq c_{1}\left(\beta_{0}, \beta_{1}, \beta_{2}, \beta_{3}, \gamma_{0}\right)\left(1+c_{\chi}\right)^{2} \int_{\Omega_{\tau}}\left[\beta_{4}^{2} \Lambda_{0} \zeta^{2}+\lambda_{0} a^{i j} D_{i} \zeta D_{j} \zeta\right] \chi d \mu .
\end{aligned}
$$

Proof. If $\zeta$ vanishes on $\Gamma$, the estimate follows from the same arguments as in [12, Lemma 4.7; 21, Lemma 3.1] except for a slight modification concerning the boundary integrals. By using $\eta=\left(v_{1}-\tau\right)_{+} \chi\left(v_{1}\right) \zeta^{2}(A+\overline{\varphi \gamma})$ in [21, Lemma $1.1]$, we are led to examine the integral

$$
I=\int_{\partial \Omega}\left[D_{k} \varphi+\varphi_{z} D_{k} u+A \cdot \delta_{T} \gamma_{k}-\gamma_{i} D_{k}^{i}\right]\left(A^{k}+\overline{\varphi \gamma}^{k}\right)\left(v_{1}-\tau\right)_{+} \chi\left(v_{1}\right) \zeta^{2} d s .
$$

Since $D_{i} \gamma_{k}=D_{k} \gamma_{i}$,

$$
\begin{aligned}
D_{k} \varphi+\varphi_{z} D_{k} u & =D_{k}(\bar{\varphi} \gamma \cdot \bar{\gamma})+\bar{\varphi}_{z} \gamma \cdot \bar{\gamma} D_{k} u \\
& =\gamma \cdot \bar{\gamma}\left[D_{k} \bar{\varphi}+\bar{\varphi}_{z} D_{k} u\right]+\bar{\gamma} \cdot D_{k} \gamma \bar{\varphi}+\left(D_{k} \bar{\gamma}\right) \cdot \gamma \bar{\varphi},
\end{aligned}
$$

and $\gamma \cdot(A+\overline{\varphi \gamma})=0$ on $\partial \Omega$, we have

$$
\begin{aligned}
I= & \int_{\partial \Omega}(A+\overline{\varphi \gamma}) \cdot \delta_{T} \gamma_{k}\left(A^{k}+\overline{\varphi \gamma}^{k}\right)\left(v_{1}-\tau\right)_{+} \chi\left(v_{1}\right) \zeta^{2} d s \\
& +\int_{\partial \Omega}\left[\gamma \cdot \bar{\gamma}\left\{D_{k} \bar{\varphi}+\bar{\varphi}_{z} D_{k} u\right\}+\left(\bar{\varphi} \gamma \cdot D_{k} \bar{\gamma}\right)\right]\left(A^{k}+\overline{\varphi \gamma}^{k}\right)\left(v_{1}-\tau\right) \chi\left(v_{1}\right) \zeta^{2} d s \\
& -\int_{\partial \Omega} \gamma_{i} D_{k}^{i}\left(A^{k}+\bar{\varphi} \gamma^{k}\right)\left(v_{1}-\tau\right)_{+} \chi\left(v_{1}\right) \zeta^{2} d s \\
\leq & c_{2}\left(n, \beta_{1}\right) \beta_{4} \int_{\partial \Omega} \lambda_{0} \Lambda_{1}\left(1-\frac{\tau}{v_{1}}\right)_{+} \chi \zeta^{2} v d s .
\end{aligned}
$$

Using Lemma 1.2 and [12, $(4.11 \mathrm{c})]$ in its more precise form

$$
\lambda_{0} a^{i j} D_{i k} u D_{j k} u \leq K_{1}\left(\beta_{1}, \beta_{2}, \beta_{3}\right)\left(\mathscr{C}^{2}+\mathscr{E}+\beta_{4}^{2} \Lambda_{0}\right) v^{2}
$$

gives, for any $\eta>0$,

$$
\begin{aligned}
I \leq & \eta \int_{\Omega_{\tau}}\left[\left(1-\frac{\tau}{v_{1}}\right) \mathscr{C}^{2}+\mathscr{E}\right] \chi d \mu \\
& +c_{3}\left(n, \beta_{0}, \beta_{1}, \beta_{2}, \beta_{3}, \gamma_{0}\right) \frac{1}{\eta}\left(1+c_{\chi}\right)^{2} \int_{\Omega_{\tau}}\left[\beta_{4}^{2} \Lambda_{0} \zeta^{2}+\lambda_{0} a^{i j} D_{i} \zeta D_{j} \zeta\right] \chi d \mu
\end{aligned}
$$

just as on p. 238 of [12]. This estimate is just what is needed to prove (3.14) if $\zeta$ vanishes on $\Gamma$. 
For general $\zeta$, we first replace $\zeta$ by $\theta_{k} \zeta$ to obtain

$$
\begin{aligned}
\int_{\Omega_{\tau}}[ & \left.\left(1-\frac{\tau}{v_{1}}\right) \mathscr{C}^{2}+\mathscr{E}\right] \chi \theta_{k}^{2} \zeta^{2} d \mu \\
\leq & 2 c_{1}\left(1+c_{\chi}\right)^{2} \int_{\Omega_{\tau}}\left[\beta_{4}^{2} \Lambda_{0} \zeta^{2}+\lambda_{0} a^{i j} D_{i} \zeta\right] \chi \theta_{k}^{2} d \mu \\
& +2 c_{1}\left(1+c_{\chi}\right)^{2} \int_{\Omega_{\tau}} \lambda_{0} a^{i j} D_{i} \theta_{k} D_{j} \theta_{k} \chi \zeta^{2} d \mu .
\end{aligned}
$$

By hypothesis, the right-hand side is bounded, independent of $k$, by some constant $K_{2}$ (which does depend on the gradient bound and on the specific function $\chi$ ). Thus, for any compact subset $S$ of $\bar{\Omega} \backslash \Gamma$, we have

$$
\int_{\Omega_{\tau}}\left[\left(1-\frac{\tau}{v_{1}}\right) \mathscr{C}^{2}+\mathscr{E}\right] \chi I_{s} \theta_{k}^{2} \zeta^{2} d \mu \leq K_{2}
$$

where $I_{s}=1$ on $S$ and $=0$ off of $S$. Sending $k \rightarrow \infty$ allows us to replace $\theta_{k}$ by 1 in this inequality. Then choosing a sequence of $S$ 's which expand to $\Omega$ yields

$$
\int_{\Omega_{\tau}}\left[\left(1-\frac{\tau}{v_{1}}\right) \mathscr{C}^{2}+\mathscr{E}\right] \chi \zeta^{2} d \mu \leq K_{2} .
$$

Since this integral is finite, the proof of (3.14) given for $\zeta$ vanishing on $\Gamma$ applies for arbitrary $\zeta$.

Before proceeding to our gradient estimate, we prove a Sobolev inequality which involves several of our structure conditions. We remark that a much simpler form of this inequality can be used if $\partial \Omega \in C^{2}$ (see [12, Lemmata 1.3 and 2.1(b)]).

Lemma 3.2. Let $h \in W^{1,2} \cap L^{\infty}$ be nonnegative and suppose $h=0$ anywhere that $v_{1}<\tau_{0}$. Suppose also that structure conditions (3.4), (3.5b, c, e, f), (3.6), $(3.8 \mathrm{e}, \mathrm{g}, \mathrm{h})$ are satisfied. Then

$$
\begin{aligned}
\int_{\Omega} h^{2(n+2) / n} d \mu \leq & c_{4}\left(\beta_{0}, \beta_{1}, \beta_{2}, \beta_{3}, \gamma_{0}, n\right)\left(\int_{\Omega} h^{2} d \mu\right)^{2 / n} \\
& \times\left(\int_{\Omega}\left[|\delta h|^{2}+h^{2}\left(\mathscr{C}^{2}+\mathscr{E}+\beta_{4}^{2} \Lambda_{0}\right) / \lambda\right] d \mu\right) .
\end{aligned}
$$

Proof. As in [12, Lemma 1.3], it follows from [25, p. 372; 13, Lemma 1.1] that if the nonnegative numbers $p, q, r$ satisfy $\frac{p}{n}+r+1=q$, then

$$
\begin{aligned}
\int_{\Omega} h^{q} d \mu \leq & c_{5}(p, q, r)\left(\int_{\Omega} h^{p} d \mu\right)^{1 / 2} \\
& \times\left(\int_{\Omega}\left[h^{r}|\delta h|+h^{r+1}\left|\frac{1}{n v} g^{i j} D_{i j} u\right|\right] d \mu+\int_{\partial \Omega} h^{r+1} v d s\right) .
\end{aligned}
$$

Now we use Lemma 1.2 with $h$ replaced by $h^{r+1}$ to estimate the boundary 
integral. We have

$$
\begin{aligned}
D u \cdot & \bar{\gamma} D_{i}\left(\frac{A^{i}}{|A|}\right)-\bar{\gamma}^{i} D_{j} u D_{i}\left(\frac{A^{j}}{|A|}\right) \\
= & \frac{1}{|A|} a^{i k} D_{j k} u\left(\delta_{i}^{j} \nu \cdot \bar{\gamma}-\bar{\gamma}^{j} \nu_{i}\right)-\frac{1}{|A|^{3}} a^{i k} D_{j k} u\left(\nu \cdot A A^{i} \bar{\gamma}^{j}-\nu \cdot \bar{\gamma} A^{i} A^{j}\right) \\
& \quad+\frac{1}{|A|}\left(\bar{\gamma}^{i} D_{i} u A_{j}^{j}-\bar{\gamma}^{j} D_{i} u A_{j}^{i}\right)-\frac{1}{|A|^{3}}\left(D u \cdot \bar{\gamma} A^{k} A_{j}^{k} A^{j}-D u \cdot A \bar{\gamma}^{j} A^{k} A_{j}^{k}\right) \\
\leq & c_{6}\left(n, \beta_{1}, \beta_{2}, \beta_{3}\right)\left[\left(a^{i j} D_{i k} u D_{j k} u\right)^{1 / 2}\left(\frac{\bar{\mu}}{\lambda_{0}^{2}}\right)^{1 / 2}+\beta_{4}\left(\frac{\Lambda_{0}}{\bar{\mu} \lambda}\right)^{1 / 2} v\right] \\
\leq & c_{7}\left(n, \beta_{1}, \beta_{2}, \beta_{3}\right)\left[\left(\mathscr{C}^{2}+\mathscr{E}+\beta_{4}^{2} \Lambda_{0}\right) / \lambda\right]^{1 / 2} v
\end{aligned}
$$

by $(3.4),(3.5 \mathrm{c})$, and $(3.8 \mathrm{e}, \mathrm{g}, \mathrm{h}),(3.15)$. Hence Lemma 1.2 implies that

$$
\begin{aligned}
\int_{\partial \Omega} & h^{r+1} v d s \leq c_{8}\left(\beta_{3}, \gamma_{0}\right) \int_{\partial \Omega} h^{r+1} D u \cdot \frac{A}{|A|} \gamma \cdot \bar{\gamma} d s \\
& \leq c_{9}\left(\beta_{1}, \beta_{2}, \beta_{3}, \gamma_{0}, n\right) \int_{\Omega}\left\{r h^{r}|\delta h|+h^{r+1}\left[\left(\mathscr{C}^{2}+\mathscr{E}+\beta_{4}^{2} \Lambda_{0}\right) / \lambda\right]^{1 / 2}\right\} d \mu .
\end{aligned}
$$

Observing from $(3.5 b)$ that

$$
\lambda\left|\frac{1}{n v} g^{i j} D_{i j} u\right|^{2} \leq \frac{\lambda_{0}}{v^{2}}\left(a^{i j} D_{i k} u D_{j k} u\right),
$$

we see that

$$
\begin{aligned}
\int_{\Omega} h^{q} d \mu \leq & c_{10}\left(\beta_{1}, \beta_{2}, \beta_{3}, \gamma_{0}, n, p, r\right)\left(\int_{\Omega} h^{p} d \mu\right)^{1 / n} \\
& \times \int_{\Omega}\left[h^{r}|\delta h|+h^{r+1}\left\{\left(\mathscr{C}^{2}+\mathscr{E}+\beta_{4}^{2} \Lambda_{0}\right) / \lambda\right\}^{1 / 2}\right] d \mu .
\end{aligned}
$$

This inequality is just $[13,(1.3)]$ with $|H|$ replaced by $\left.\left\{\left(\mathscr{C}^{2}+\mathscr{E}+\beta_{4}^{2} \Lambda_{0}\right)\right] \lambda\right\}^{1 / 2}$, so the proof is completed by choosing $q=2(n+2) / n, p=1+q / 2$, and $r=$ $1+2 / n-2 / n^{2}$, and then applying Schwarz's inequality and Hölder's inequality as on $[13$, p. 581$]$.

From this point, the proof of our gradient bound proceeds as in [12] with the obvious changes (for example, Lemma 3.2 in place of [12, Lemma 1.3] and $\sup _{B_{\rho} \cap \Omega} u-u$ in place of $u-\inf u$ in the proof of Lemma 3.5), the sharper form of the calculations in [21], and the observation that our (3.3a), and $(3.5 \mathrm{~b}, \mathrm{e}, \mathrm{f})$ can be used in place of $[12,(4.11 \mathrm{e})]$. For this reason we only state the results.

Lemma 3.3. Suppose conditions (3.2a, c) and (3.3)-(3.8) are satisfied. Then for any $x_{0} \in \Omega, \tau \geq \tau_{0}$, and $\rho>0$, we have

$$
\begin{aligned}
& \sup _{\Omega_{\tau, \rho / 2}}\left\{\left(1-\frac{\tau}{v_{1}}\right)^{n+2} w^{2}\right\} \\
& \quad \leq c_{11}\left(\beta, \beta_{1}, \beta_{2}, \beta_{3}, \beta_{4} \rho, \gamma_{0}, n\right) \rho^{-n} \int_{\Omega_{\tau, \rho}} w^{2}\left(\frac{\Lambda}{\lambda}\right)^{(n+2) / 2} v d x .
\end{aligned}
$$


Lemma 3.4. Suppose conditions (3.2b, c) (3.3a, c) (3.4), (3.5a, c, d, e), (3.6), (3.7), (3.8), and (3.10) are satisfied, let $q>0$, and set

$$
\sigma=\underset{\Omega \cap B_{\rho}}{\operatorname{osc}} u \text {. }
$$

Then there is a constant $c_{12}=c_{12}\left(\beta, \beta_{1}, \beta_{2}, \beta_{3}, \beta_{7}, \gamma_{0}, n, q\right)$ such that if there is $a \tau_{1} \geq \tau$ for which

$$
8\left(c_{12} \beta_{4}^{2}+\beta_{8}^{2}\right) \sigma^{2} \varepsilon\left(\tau_{1}\right) \leq 1,
$$

then

$$
\begin{aligned}
\int_{\Omega_{\tau, \rho / 2}} w^{q} D u \cdot A d x \leq & c_{13}\left(\beta, \beta_{0}, \beta_{1}, \beta_{2}, \beta_{3}, \beta_{4} \rho, \beta_{7}, \beta_{8} \rho, \gamma_{0}, n, q\right) \\
& \times\left[w\left(\tau_{1}\right)+\frac{\sigma}{\rho}\right]^{q} \int_{\Omega_{\tau, \rho}} D u \cdot A d x .
\end{aligned}
$$

For our next lemma, we introduce the quantities

$$
\Omega_{\tau}^{\prime}=\{x \in \Omega: v(x)>\tau\}, \quad \Omega_{\tau, \rho}^{\prime}=\Omega_{\tau}^{\prime} \cap B_{\rho}, \quad K(\rho)=\rho^{1-n}\left|B_{\rho} \cap \partial \Omega\right| .
$$

Lemma 3.5. Suppose conditions (3.12a, b) hold on $\Omega_{\tau}^{\prime}$ and define $\sigma$ by (3.18). Then for any $\rho>0, x_{0} \in \Omega$, and $\tau_{2} \geq \tau$ such that

$$
\varepsilon_{1}\left(\tau_{2}\right) \leq \frac{\rho}{8 \sigma}
$$

and

$$
\Delta_{1}=\sup _{\substack{x \in B_{\rho} \\ v \leq \tau_{2}}}\left\{\sigma\left(B+\beta_{9} D u \cdot A\right)_{-}+(D u \cdot A)_{-}+\frac{4 \sigma|A|}{\rho}\right\},
$$

we have

$$
\int_{\Omega_{\tau, \rho / 2}^{\prime}} D u \cdot A d x \leq c_{14}(n) \exp \left(\beta_{9} \sigma\right) \rho^{n}\left(K(\rho) \frac{\sigma}{\rho}+\Delta_{1}\right) .
$$

Although Lemmata 3.3-3.5 are readily combined to give a gradient bound, we shall not do so here. The details are generally straightforward but, as we shall see in $\S 5$, it is sometimes advantageous to use only the estimates from Lemmata 3.3 and 3.5 .

As in $[28, \S 3]$ (and $[21, \S 4]$ ), the form of our estimates can be improved if the structure conditions of $\S 3$ are appropriately strengthened. Since these estimates are proved by modifying the proofs of $\S 3$ in exactly the way discussed in [28, 21 ], we state them without proof.

Theorem 3.6. Let $x_{0} \in \Omega, \rho>0$, and suppose conditions (3.1)-(3.10) hold with $\varepsilon\left(v_{1}\right)=\beta_{10} w^{-2}$. Suppose also that

$$
B \geq-\beta_{11}|A| \text { in } \Omega_{\tau_{0}}^{\prime} .
$$

With $\sigma$ given by (3.18), set

$$
\begin{gathered}
\tau_{3}=\max \left\{\tau_{0}, \frac{8 \sigma}{\beta_{3} \rho}+2 \frac{\beta_{11} \sigma}{\beta_{3}}\right\}, \\
\Delta_{2}=\sup _{|D u| \leq \tau_{3}}\left\{\sigma B_{-}+(D u \cdot A)_{-}+\frac{\sigma}{\rho}|A|\right\} .
\end{gathered}
$$


Then there is a constant $c_{15}=c_{15}\left(\beta, \beta_{0}, \beta_{1}, \beta_{2}, \beta_{3}, \beta_{4} \rho, \beta_{5}, \beta_{7}, \beta_{8} \rho, \beta_{10}\right.$, $\left.\beta_{11} \rho, \gamma_{0}, K(\rho), n\right)$ such that

$$
\sup _{\Omega_{\tau, \rho / 8}}\left(1-\frac{\tau_{0}}{v_{1}}\right)_{+}^{n+2} w^{2} \leq c_{15}\left[w\left(\tau_{0}\right)+\frac{\sigma}{\rho}\right]^{2+\beta_{6}}\left(\Delta_{2}+\varphi_{0} \frac{\sigma}{\rho}\right) .
$$

If also

$$
a^{i j} \xi_{i} \eta_{j} \leq \beta_{12}\left\{\Lambda_{0} g^{i j} \eta_{i} \eta_{j}\right\}^{1 / 2}\left\{a^{i j} \xi_{i} \xi_{j}\right\}^{1 / 2}
$$

for all $\eta, \xi$ in $\mathbb{R}^{n}$, set

$$
\begin{gathered}
\sigma_{0}=\sup _{\Omega \cap B_{\rho}}\left(u-u\left(x_{0}\right)\right)_{+}, \\
\tau_{4}=\max \left\{\tau_{0}, \frac{8 \sigma_{0}}{\beta_{3} \rho}+\frac{2 \beta_{11}}{\beta_{3}} \sigma_{0}\right\}, \\
\Delta_{3}=\sup _{\substack{|D u| \leq \tau_{4} \\
u-u\left(x_{0}\right) \geq \rho}}\left\{\sigma_{0} B_{-}+(D u \cdot A)_{-}+\frac{\sigma_{0}}{\rho}|A|\right\} .
\end{gathered}
$$

Then there is $c_{16}=c_{16}\left(\beta, \beta_{0}, \beta_{1}, \beta_{2}, \beta_{3}, \beta_{4} \rho, \beta_{5}, \beta_{7}, \beta_{8} \rho, \beta_{10}, \beta_{11} \rho, \beta_{12}\right.$, $\left.\gamma_{0}, K(\rho), n\right)$ such that

$$
\sup _{\Omega_{\tau, \rho / 8}}\left(1-\frac{\tau_{0}}{v_{1}}\right)_{+}^{n+2} w^{2} \leq c_{16}\left[1+w\left(\tau_{0}\right)\right]\left[1+w\left(\tau_{0}\right)+\frac{\sigma}{\rho}\right]^{1+\beta_{6}}\left(\Delta_{3}+\varphi_{0} \frac{\sigma}{\rho}\right) .
$$

\section{THE PARABOLIC CASE}

Parabolic versions of the results in $\S 3$ are also valid. We only state analogs of Lemmata 3.3, 3.4, and 3.5 including a version which allows localization in time as well as space. Refined estimates are then easy to obtain (see [21, $\S 4]$ ).

To state these estimates, we introduce some additional terminology: For $T>0$, we set

$$
Q_{T}=\Omega \times(0, T), \quad S Q_{T}=\partial \Omega \times(0, T),
$$

and we consider the problem

$$
\begin{aligned}
\operatorname{div} A(x, t, u, D u)+B(x, t, u, D u) & =u_{t} & & \text { in } Q_{T}, \\
A(x, t, u, D u) \cdot \gamma+\varphi(x, t, u) & =0 & & \text { on } S Q_{T}, \\
u(\cdot, 0) & =u_{0} & & \text { in } \Omega
\end{aligned}
$$

for some known function $u_{0}$. (Here $D u$ is only the spatial gradient of $u$.) Next we define

$$
\begin{aligned}
& Q(\tau)=\left\{(x, t) \in Q_{T}: v_{1}(x, t)>\tau\right\}, \\
& Q(\tau, \rho)=\left\{(x, t) \in Q(\tau): x \in B_{\rho}\right\}, \\
& S Q(\tau)=\left\{(x, t) \in S Q_{T}: v_{1}(x, t)>\tau\right\}
\end{aligned}
$$

Moreover all structure conditions are assumed to hold with $Q\left(\tau_{0}\right)$ replacing $\Omega_{\tau_{0}}$ and $S Q\left(\tau_{0}\right)$ replacing $\Gamma_{\tau_{0}}$. 
Since $A, \varphi$, and $F$ may depend on $t$, we assume the following bounds:

$$
\begin{gathered}
\bar{\varphi}_{t} \bar{\gamma} \cdot D u+F_{t} \leq \beta_{4}^{2} \Lambda_{0}, \\
\bar{\mu} F_{z}^{2} \leq \beta_{4}^{2} \lambda_{0} \Lambda_{0}, \\
B F_{z} \leq \beta_{4}^{2} \Lambda_{0}, \\
\left(v\left|A_{z}\right|+\left|A_{x}\right|\right)\left|F_{z}\right| \leq \beta_{4}^{2} \Lambda_{0}, \\
\lambda \leq \lambda_{0} v,
\end{gathered}
$$

and we suppose that

$$
\xi^{-\beta} \lambda_{0} \quad \text { is decreasing. }
$$

Lemma 4.1. Let $\chi$ be a nonnegative Lipschitz function on $\left[\tau_{0}, \infty\right)$, let $\tau \leq \tau_{0}$, and suppose there is a constant $c_{\chi}$ for which (3.14) holds. If $\zeta \in C^{0,1}\left(Q_{T}\right)$, if $\zeta(x, 0)=0$ in $\Omega$ or if $v_{1}(x, 0) \leq \tau$ in $\Omega$, and if conditions (3.3a, c), (3.4), (3.5a, c, d, e), (3.6), (3.7), (3.8), (4.1), and (4.3) hold, then there is a constant $c_{17}=c_{17}\left(\beta, \beta_{0}, \beta_{1}, \beta_{2}, \beta_{3}, \gamma_{0}, n\right)$ such that

$$
\sup _{0<0<T} \int_{\Omega \times\{t\}}\left(v_{1}-\tau\right)_{+}^{2} \lambda_{0} \chi \zeta^{2} d x+\int_{0}^{T} \int_{\Omega_{\tau}}\left[\left(1-\frac{\tau}{v_{1}}\right) \mathscr{C}^{2}+\mathscr{E}\right] \chi \zeta^{2} d \mu d t
$$

$$
\begin{aligned}
\leq c_{17}\left(1+c_{\chi}\right)^{3}\left\{\int_{0}^{t} \int_{\Omega_{\tau}}\left[\beta_{4}^{2} \Lambda_{0} \zeta^{2}+\lambda_{0} a^{i j} D_{i} \zeta D_{j} \zeta\right] \chi d \mu d t\right. & \\
& \left.+\int_{0}^{T} \int_{\Omega_{\tau}}\left(v_{1}-\tau\right)^{2} \chi\left|\zeta_{t}\right| \zeta d x d t\right\} .
\end{aligned}
$$

Proof. From the proof of Lemma 3.1, we find that

$$
\begin{aligned}
\int_{0}^{h} \int_{\Omega_{\tau}} & {\left[\left(1-\frac{\tau}{v_{1}}\right) \mathscr{C}^{2}+\mathscr{E}\right] \chi \zeta^{2} d \mu d t } \\
\leq & c_{1}\left(1+c_{\chi}\right)^{2} \int_{0}^{h} \int_{\Omega_{\tau}}\left[\beta_{4}^{2} \Lambda \zeta^{2}+\lambda_{0} a^{i j} D_{i} \zeta D_{j} \zeta\right] \chi d \mu d t \\
& +c_{18}\left(c_{1}\right) \int_{0}^{h} \int_{\Omega} u_{t} D_{k}\left[\left(A^{k}+\overline{\varphi \gamma}^{k}\right)\left(v_{1}-\tau\right) \chi \zeta^{2}\right] d x d t
\end{aligned}
$$

for any $h \in(0, T)$. Thus it suffices to estimate this last integral, which we denote by $I$.

Setting

$$
\Xi(s)=\int_{\tau}^{s} \frac{(\sigma-\tau)+\chi(\sigma)}{v_{1}^{\prime}\left(v_{1}^{-1}(\sigma)\right)} d \sigma
$$

and using the approximation and integration by parts argument from [13, Lemma 3.1] yields

$$
\begin{aligned}
I= & -\int_{\Omega \times\{h\}} \Xi\left(v_{1}\right) \zeta^{2} d x+2 \int_{0}^{h} \int_{\Omega_{\tau}} \Xi\left(v_{1}\right) \zeta \zeta_{t} d x d t \\
& +\int_{0}^{h} \int_{\Omega_{\tau}} u_{t} F_{z}\left(v_{1}-\tau\right) \chi \zeta^{2} d x d t \\
& +\int_{0}^{h} \int_{\Omega_{\tau}}\left(F_{t}+\bar{\varphi}_{t} \bar{\gamma} \cdot D u\right)\left(v_{1}-\tau\right) \chi \zeta^{2} d x d t .
\end{aligned}
$$


By using $[13,(3.6)]$ with $\chi \lambda_{0}$ replacing $\chi$, we see from (4.3) and (3.5d) that

$$
\frac{\beta_{1}}{2} \lambda_{0} \chi\left(v_{1}-\tau\right)^{2} \geq \Xi \geq \frac{1}{2\left(1+\beta+c_{\chi}\right)} \lambda_{0} \chi\left(v_{1}-\tau\right)^{2} .
$$

We use this inequality to estimate the first two integrals in (4.5). In the third integral, we use the differential equation in the form

$$
u_{t}=\frac{1}{v} a^{i j} D_{i j} u+B+A_{z}^{i} D_{i} u+A_{i}^{i} .
$$

Thus

$$
\begin{aligned}
I \leq & -\frac{1}{2\left(1+\beta+c_{\chi}\right)} \int_{\Omega \times\{h\}}\left(v_{1}-\tau\right)^{2} \lambda_{0} \chi \zeta^{2} d x+\beta_{0} \int_{0}^{h} \int_{\Omega_{\tau}}\left(v_{1}-\tau\right)^{2} \chi\left|\zeta_{t}\right| \zeta d x d t \\
& \left.+\int_{0}^{h} \int_{\Omega_{\tau}}\left(1-\frac{\tau}{v_{1}}\right) \frac{\lambda_{0}}{v^{2}} a^{i j} D_{i k} u D_{j k} u\right]^{1 / 2}\left[\frac{\bar{\mu}}{\lambda_{0}} F_{z}^{2}\right]^{1 / 2} \chi \zeta^{2} d \mu d t \\
& +\int_{0}^{h} \int_{\Omega_{\tau}}\left[B F_{z}+\left(v\left|A_{z}\right|+\left|A_{x}\right|\right) F_{z}\right] \chi \zeta^{2} d \mu d t \\
& +\int_{0}^{h} \int_{\Omega_{\tau}}\left(F_{t}+\bar{\varphi}_{t} \bar{\gamma} \cdot D u\right) \chi \zeta^{2} d \mu d t .
\end{aligned}
$$

If we estimate these last three integrals via (4.1), Schwarz's inequality, and (3.16), we obtain (4.4).

Using Lemma 4.1 in place of Lemma 3.1 gives analogs of Lemmata 3.3, 3.4, 3.5 by virtue of the proofs of [21, Lemmata 3.2, 3.3, 3.4, Theorem 4.1].

Lemma 4.2. Suppose conditions (3.2a, c), (3.3)-(3.8), (4.1), (4.2), and (4.3) are satisfied, and let $x_{0} \in \Omega$, and $\rho>0$.

(a) If $v_{1} \leq \tau_{0}$ on $\Omega \times\{0\}$, then

$$
\begin{gathered}
\sup _{Q(\tau, \rho / 2)}\left(1-\frac{\tau}{v_{1}}\right)^{n+2} w^{2} \leq c_{19}\left(\beta, \beta_{0}, \beta_{1}, \beta_{2}, \beta_{3}, \beta_{4} \rho, \gamma_{0}, n\right) \\
\times \rho^{-n-2} \iint_{Q(\tau, \rho)} w^{2}\left(\frac{\Lambda}{\lambda}\right)^{(n+2) / 2} v d x d t
\end{gathered}
$$

(b) If also

$$
\lambda_{0} v \leq \beta_{13} \Lambda,
$$

and $\rho^{2}<t_{0}<T$, then

$$
\begin{aligned}
\left(1-\frac{\tau}{v_{1}}\right)_{+}^{n+2} w^{2}\left(x_{0}, t_{0}\right) \leq c_{20}( & \left.\beta, \beta_{0}, \beta_{1}, \beta_{2}, \beta_{3}, \beta_{4} \rho, \beta_{13}, \gamma_{0}, n\right) \\
& \times \rho^{-n-2} \int_{t_{0}-\rho^{2}}^{t_{0}} \int_{\Omega_{\tau, \rho}} w^{2}\left(\frac{\Lambda}{\lambda}\right)^{(n+2) / 2} v d x d t
\end{aligned}
$$

Lemma 4.3. Suppose conditions (3.2b, c), (3.3a, c), (3.4), (3.5a, c, d, e), (3.6), (3.7), (3.8), (3.10), (4.1), (4.2), and (4.3) are satisfied, and let $q>0, x_{0} \in \Omega$, and $\rho>0$. 
(a) If $v_{1} \leq \tau$ on $\Omega \times\{0\}$, set

$$
\sigma=\sup \left\{\left|u(x, t)-u\left(x_{0}, t\right)\right|: x \in B_{\rho}, 0<t<T\right\} .
$$

Then there is a constant $c_{21}=c_{21}\left(\beta, \beta_{1}, \beta_{2}, \beta_{3}, \beta_{7}, \gamma_{0}, n, q\right)$ such that if there is a $\tau_{1} \geq \tau$ for which

$$
8\left(c_{21} \beta_{4}^{2}+\beta_{8}^{2}\right) \sigma^{2} \varepsilon\left(\tau_{1}\right) \leq 1
$$

then

$$
\begin{aligned}
& \iint_{Q(\tau, \rho / 2)} w^{q} D u \cdot A d x d t \\
& \leq c_{22}\left(\beta, \beta_{0}, \beta_{1}, \beta_{2}, \beta_{3}, \beta_{4} \rho, \beta_{7}, \beta_{8} \rho, \gamma_{0}, n, q\right) \\
& \quad \times\left[w\left(\tau_{1}\right)+\frac{\sigma}{\rho}\right]^{q} \iint_{Q(\tau, \rho)} D u \cdot A d x d t
\end{aligned}
$$

(b) If also

$$
v^{2} \leq \beta_{14} w D u \cdot A
$$

and $\rho^{2}<t_{0}<T$, set

$$
\sigma=\sup \left\{\left|u(x, t)-u\left(x_{0} t\right)\right|: x \in B_{\rho}, t_{0}-\rho^{2}<t<t_{0}\right\} .
$$

If there is a $\tau_{1} \geq \tau$ for which (4.10) holds, then there is a constant $c_{23}=$ $c_{23}\left(\beta, \beta_{0}, \beta_{1}, \beta_{2}, \beta_{3}, \beta_{4} \rho, \beta_{7}, \beta_{8} \rho, \gamma_{0}, n, q\right)$ such that

$$
\begin{aligned}
& \int_{t_{0}-\rho^{2} / 4}^{t_{0}} \int_{\Omega_{\tau, \rho / 2}} w^{q} D u \cdot A d x d t \\
& \quad \leq c_{23}\left[w\left(\tau_{1}\right)+\frac{\sigma}{\rho}+\beta_{14}\left(\frac{\sigma}{\rho}\right)^{2}\right] \int_{t_{0}-\rho^{2}}^{t_{0}} \int_{\Omega_{\tau, \rho}} D u \cdot A d x d t .
\end{aligned}
$$

For our final lemma, we define

$$
\begin{gathered}
Q^{\prime}(\rho)=\left\{(x, t) \in Q_{T}: x \in B_{\rho}, t_{0}-\rho^{2}<t<t_{0}\right\}, \\
Q^{\prime}(\rho, \tau)=\left\{(x, t) \in Q^{\prime}(\rho): v(x, t) \geq \tau\right\} .
\end{gathered}
$$

Lemma 4.4. Let $\tau \geq 1, \rho>0, x_{0} \in \Omega$, and $0<t_{0}<T$. Suppose conditions (3.11) hold on $Q^{\prime}(\rho, \tau)$ and set

$$
M=\underset{Q^{\prime}(\rho)}{\operatorname{osc}} u
$$

If $\tau_{5} \geq \tau$ is so large that

$$
\varepsilon_{1}\left(\tau_{5}\right) \leq \frac{\rho}{8 M}
$$

and if

$$
\Delta_{4}=\sup _{Q^{\prime}(\rho) \backslash Q^{\prime}(\rho, \tau)}\left\{M\left(B+\beta_{9} D u \cdot A\right)_{-}+(D u \cdot A)_{-}+\frac{M}{\rho}|A|\right\},
$$

then

(4.18)

$\iint_{Q^{\prime}(\rho / 2, \tau)} D u \cdot A d x d t \leq c_{24}(n) \exp \left(\beta_{9} M\right) \rho^{n}\left[M^{2}+\Delta_{4} \rho^{2}+K(\rho) M \rho \varphi_{0}\right]$ 
Note that Lemma 4.4 is identical to [21, Lemma 3.4].

\section{EXAMPLES}

Our structure conditions, which are based on those in [28], were chosen to include all the examples in [21] and hence all those in [28, 12, 30] as well. Rather than reproduce them all here, we only include three to show the range of these methods.

Example 1 (The capillary problem). Suppose

$$
A=\nu, \quad B=n H(x, u, D u)+b(x, u, D u)
$$

for Lipschitz $H$ with

$$
\begin{array}{cc}
n H \geq-\theta_{1}, & v|b| \leq \theta_{1}, \\
n v\left[\left|H_{p}\right|+\left|H_{p} \cdot p\right|\right] \leq \theta_{1}, & n\left[v H_{z}+\left|H_{x}\right|\right] \leq \theta_{1}^{2}, \\
\varphi_{0}<1, & \varphi_{z} \leq 0 .
\end{array}
$$

We then set

$$
\begin{gathered}
C_{k}^{i}=b \delta_{k}^{i}, \quad D_{k}^{i}=n H \delta_{k}^{i}, \quad v_{1}=\frac{1}{2}(v+\varphi D u \cdot \gamma), \\
\underline{\mu}=v^{-2}, \quad w=\log v_{1}, \quad \lambda^{1 / 3}=\lambda_{0}=\frac{1}{2}\left(1-\varphi_{0}\right), \quad \bar{\mu}=\Lambda=\Lambda_{0}=\Lambda_{1}=1 .
\end{gathered}
$$

With these choices,

$$
\begin{gathered}
\beta=1, \quad \beta_{4}=c\left(\varphi_{0}\right)\left(\theta_{1}+\frac{1}{R}\right), \quad \beta_{6}=0, \quad \beta_{7}=2, \\
\beta_{8}=0, \quad \beta_{11}=c\left(\varphi_{0}\right) \theta_{1},
\end{gathered}
$$

and $\tau_{0}, \beta_{0}, \ldots, \beta_{3}, \beta_{5}, \beta_{10}$ chosen suitably (depending only on $\varphi_{0}$ and $n$ ), Theorem 3.6 gives an estimate of the form

$$
v\left(x_{0}\right) \leq c_{1} \exp \left(c_{2} \sigma_{0} / \rho\right)
$$

with $c_{1}$ and $c_{2}$ depending only on $\theta_{1} \rho, K(\rho), n, \gamma_{0}, \varphi_{0}$, and $\rho / R$. (Recall that $R$ is the radius of the exterior sphere.) Because of the restrictions on $\varphi$ forced by the inequality $\varphi_{0}<1$, this estimate is a sharp local version of [ 9 , Lemma 4.5].

Let us examine briefly the special case that $\Gamma$ is an edge from $[9$, Theorem 4.2]. Then, at least locally, $\Gamma$ is the intersection of two $C^{2}$ hypersurfaces $S_{1}$ and $S_{2}$ with inner normals $\gamma_{1}$ and $\gamma_{2}$. Let $\theta$ denote the angle between $S_{1}$ and $S_{2}$ along $\Gamma$; by the exterior sphere condition $\theta<\pi$. If $\varphi=\cos \beta$ is Lipschitz with $|\pi-2 \beta|<\theta$ on $\Gamma$, then an appropriate pseudonormal is $\bar{\gamma}=\frac{\left(\gamma_{1}+\gamma_{2}\right)}{\left|\gamma_{1}+\gamma_{2}\right|}$ (cf. $\left[9\right.$, p. 28]). More generally we obtain a gradient bound if the restrictions $\varphi_{1}$ and $\varphi_{2}$ of $\varphi$ to $S_{1}$ and $S_{2}$, respectively, are Lipschitz and if there are functions $\theta_{1}$, and $\theta_{2}$ such that

$$
\pi>\theta=\theta_{1}+\theta_{2}, \quad \sin \theta_{i}>\left|\varphi_{i}\right|, \quad \varphi_{1} \varphi_{2}>0 \text { or } \varphi_{1}=\varphi_{2}=0 \quad \text { on } \Gamma,
$$

so that a suitable $\bar{\gamma}$ can be constructed. The restriction that $\varphi$ not change sign on $\Gamma$ is clearly needed for the pseudonormal approach to work, but it is not mentioned in [9]. On the other hand, this restriction is not needed to conclude the Hölder continuity of the gradient for Lipschitz solutions as in [20]. Remark 4.8 from [9] is also applicable; it is generally not possible to construct a pseudonormal near singular sets of higher codimension. 
Example 2 (Uniformly elliptic problems). Here we suppose there are nonnegative functions $\varepsilon$ and $\psi$, with $\varepsilon$ decreasing, $\varepsilon \xi$ increasing, $\varepsilon \rightarrow 0$ at infinity, $\psi \in C^{1}(1, \infty)$, and $\psi(t)>0$ and $0 \leq t \psi^{\prime}(t) / \psi(t) \leq \theta_{0}$ for $t>1$ such that

$$
\begin{gathered}
D u \cdot A \geq v \psi(v)-\theta_{1}, \quad|A| \leq \theta_{2} \psi(v), \\
\psi(v)|\xi|^{2} \leq a^{i j} \xi_{i} \xi_{j} \leq \theta_{3} \psi(v)|\xi|^{2},
\end{gathered}
$$

$$
\left|F_{z}\right|+v^{-1}\left|F_{x}\right|+v\left|A_{z}\right|+\left|A_{x}\right|+|B| \leq \theta_{4} \varepsilon(v)^{1 / 2} v \psi(v), \quad\left|\bar{\varphi}_{x}\right|,\left|\bar{\varphi}_{z}\right| \leq \theta_{4}
$$

Let us also assume for simplicity that $\psi \rightarrow \infty$ at infinity and that $\varepsilon \psi^{2}$ is bounded away from zero. Now set $I(t)=\int_{1}^{t} \psi(s) d s$, and choose

$$
\begin{gathered}
F+\varphi D u \cdot \gamma=2 I\left(v_{1}\right), \quad D_{k}^{i}=0, \\
\underline{\mu}=\psi(v), \quad w=v_{1}, \quad \lambda_{0}=\frac{1}{2} \psi\left(v_{1}\right), \quad \lambda=\frac{1}{2}, \quad \Lambda_{0}=\frac{1}{2} \varepsilon\left(v_{1}\right)\left(v_{1} \psi\left(v_{1}\right)\right)^{2}, \\
\bar{\mu}=\theta_{3} \psi(v), \quad \Lambda=\left(v_{1} \psi\left(v_{1}\right)\right)^{2}, \quad \Lambda_{1}=c\left(\theta_{0}\right)\left(v_{1}+\psi\left(v_{1}\right) .\right.
\end{gathered}
$$

For $\beta_{4}=c(n)\left(\theta_{4}+\frac{1}{R}+|D \bar{\gamma}|\right), \tau_{0}, \beta, \beta_{0}, \ldots, \beta_{3}, \beta_{5}, \beta_{6}, \beta_{7}, \beta_{9}$ depending on $\theta_{0}, \ldots, \theta_{3}$, and $\beta_{8}=c\left(\theta_{0}, \ldots, \theta_{3}\right) \theta_{4}$, we can combine Lemmata 3.3, 3.4, and 3.5 to obtain a gradient bound of the form

$$
v\left(x_{0}\right) \leq c\left(\theta_{0}, \theta_{1}, \theta_{2}, \theta_{3}, \theta_{4} \rho, \frac{\rho}{R}, \rho|D \bar{\gamma}|, \gamma_{0}, n, \varphi_{0}, \frac{\sigma}{\rho}\right) .
$$

This estimate is also valid for degenerate equations, in which case the first inequality in $(5.1 \mathrm{c})$ can be replaced by

$$
\left|F_{z}\right|+v^{-1}\left|F_{x}\right|+v\left|A_{z}\right|+\left|A_{x}\right|+|B| \leq \theta_{4} \varepsilon(v)^{1 / 2} v \psi(v)+\theta_{5}
$$

For the nondegenerate case we have a modulus of continuity estimate from [18] (with appropriate geometry) and if $\psi$ and $v \psi$ are replaced by $(|D u|+\delta)^{m}$ and $(|D u|+1)^{m+1}$, respectively, for some $m>0$ and $\delta \in[0,1]$, then a modulus of continuity estimate follows from [10, Theorem 10.2.1]. In both cases, this modulus can be obtained (independent of $\delta$ in the second case) even for $\varepsilon=1$. By taking $\rho$ sufficiently small, we obtain a gradient bound in this case as well. ${ }^{1}$

Note that now, near an edge, the modulus of continuity estimates of [18] apply, so the only restrictions on $\varphi_{1}$ and $\varphi_{2}$ are that

$$
\varphi_{1} \varphi_{2}>0 \text { or } \varphi_{1}=\varphi_{2}=0 \text { on } \Gamma \text {. }
$$

If also $\psi(v)=v,[19$, Theorem 4.1] shows that $u$ has Hölder continuous gradient without the present gradient bounds and hence without the variational structure and without the restriction (5.2).

Example 3. Set $\Psi(v)=\exp \left(\theta v^{2}\right)$, and suppose

$$
A=\Psi(v) p, \quad|B| \leq \theta_{1} v^{2} \Psi(v), \cdot\left|\bar{\varphi}_{x}\right|+\left|\bar{\varphi}_{z}\right| \leq \theta_{1} .
$$

Then we define $v_{1}$ by

$$
\frac{1}{2 \theta} \Psi(v)+\bar{\varphi} D u \cdot \bar{\gamma}=\frac{1}{2 \theta} \Psi\left(v_{1}\right),
$$

\footnotetext{
${ }^{1}$ Note added in proof. If $\delta=t \psi^{\prime}(t) \psi(t) \leq \theta_{0}$ for some $\delta>0$, then (5.1a) and $|B| \leq$ $\theta_{4} v \psi(v)+\theta_{5}$ imply a modulus of continuity by the arguments in [31], so we can take $\varepsilon=1$ in this case also.
} 
and observe that $\left|v^{2}-v_{1}^{2}\right|$ is bounded and goes to zero as $v, v_{1} \rightarrow \infty$. Now we choose

$$
\begin{gathered}
w=\left(v_{1}\right)^{1 / 2}, \quad \underline{\mu}=\lambda_{0}=v_{1} \Psi\left(v_{1}\right), \quad \bar{\mu}=\left(1+\theta v^{2}\right) v \Psi(v), \\
\lambda=v_{1}^{5} \exp \left(\frac{n \theta}{n+2} v_{1}^{2}\right), \quad \Lambda=\Lambda_{0}=(1+\theta) v_{1}^{5} \Psi\left(v_{1}\right), \\
\Gamma_{1}=\theta_{1} v_{1} \Psi\left(v_{1}\right), \quad c_{k}^{i}=B \delta_{k}^{i}
\end{gathered}
$$

to infer that $(3.2 \mathrm{a}, \mathrm{c}),(3.3)-(3.8)$, and $(3.12 \mathrm{a})$ are satisfied with

$$
\beta=5, \quad \beta_{4}=c(n, \theta)\left(\theta_{1}+\frac{1}{R}+|D \bar{\gamma}|_{0}\right)
$$

and $\beta_{0}, \ldots, \beta_{3}, \beta_{9}$ depending only on $n$ and $\theta$ provided $\tau_{0}=c\left(\theta, \varphi_{0}\right)$. Thus Lemmata 3.3 and 3.5 along with the inequality

$$
v_{1}\left(\frac{\Lambda}{\lambda}\right)^{(n+2) / 2} v \leq c\left(\theta, n, \varphi_{0}\right) D u \cdot A
$$

imply that

$$
v\left(x_{0}\right) \leq c\left(n, \theta, \varphi_{0}, \theta_{1} \rho,|D \bar{\gamma}|_{0} \rho, K(\rho), \gamma_{0}\right) \Psi\left(\frac{\sigma}{\rho}\right) \frac{\sigma}{\rho} .
$$

Note that, near an edge, we need (5.2) to hold just as in Example 2.

We close by pointing out (see, e.g., [12, p. 250]) that we can consider a situation intermediate to the given examples, namely $A=\Psi(v) p$ for some positive $C^{1}([1, \infty))$ function $\Psi$ satisfying

$$
-1 \leq \frac{t \Psi^{\prime}(t)}{\Psi(t)} \leq \theta
$$

for $\theta>-1$ a constant and suitable conditions on $B$.

\section{OBSTACLE PROBLEMS}

We now show how to extend our estimates to solutions of obstacle problems. In particular, our results include some of those in [7] for the capillary problem (specifically Theorem 0.1 there except for the assertion of continuity of the derivatives). To state this problem, we fix a function $\psi \in C^{0,1}$ and set

$$
\mathbb{K}=\left\{v \in C^{0,1}(\overline{\mathbf{\Omega}}): v \geq \psi \text { in } \Omega\right\} .
$$

Thus we consider $u \in C^{0,1}(\bar{\Omega}) \cap W^{2,2}$ such that

$$
\int_{\Omega} A(x, u, D u) \cdot D(u-v)-B(u-v) d x \geq \int_{\partial \Omega} \varphi(u-v) d s
$$

for all $v \in \mathbb{K}$. Rather than investigate this variational inequality directly, we study the penalized problem

$$
\operatorname{div} A+B-\mu \theta_{\delta}(u-\psi)=0 \quad \text { in } \Omega, \quad A \cdot \gamma+\varphi=0 \quad \text { on } \partial \Omega
$$

for positive parameters $\mu$ and $\delta$, and $\theta_{\delta}$ a $C^{1}$ increasing function with $\theta_{\delta}(t)=0$ for $t \geq 0$ and $\theta_{\delta}(t)=-1$ for $t \leq-\delta$. 
Assuming the existence of solutions to these penalized problems, we shall bound the maxima of $|u|$ and $|D u|$ independently of $\mu$ and $\delta$. Moreover we shall show that, for any $\delta \in(0,1)$ and $\mu$ sufficiently large (depending on $\delta$ ),

$$
u-\psi \geq-C \delta^{1 / 2} \text {. }
$$

(These results are the same as [7, Theorem 1.2].) These estimates imply the regularity of the solution of the variational inequality by standard arguments.

The estimate on $u$ is simple. We have

$$
z\left[B(x, z, p)-\mu \theta_{\delta}(z-\psi)\right] \leq z B(x, z, p)
$$

for $|z| \geq|\psi|_{0}$. If $z \geq|\psi|_{0}$, this is so because $\theta_{\delta}(z-\psi)=0$ while for $z \leq 0$, we have $\theta_{\delta} \leq 0$. Hence the results of $\S 2$ gave a bound on $|u|$ independent of $\mu$ and $\delta$.

For the gradient bound, we include the term $-\mu \theta_{\delta}(z-\psi) \delta_{k}^{i}$ in $D_{k}^{i}$ and observe that we only have to check (3.8c) and (3.11a). Since

$$
\begin{gathered}
\left\{D_{i} u D_{z}\left(-\mu \theta_{\delta}(z-\psi)\right) \delta_{k}^{i}+\frac{\partial}{\partial x^{i}}\left(-\mu \theta_{\delta}(z-\psi)\right)\right\}\left\{A^{k}+\overline{\varphi \gamma}^{k}\right\} \\
=-\mu \theta_{\delta}^{\prime}(u-\psi)\left(D_{k} u-D_{k} \psi\right)\left(A^{k}+\overline{\varphi \gamma}^{k}\right) \leq 0
\end{gathered}
$$

if $v_{1} \geq \tau_{0}$ and $|D u| \geq C\left(\beta_{2}, \beta_{3}\right)|D \psi|$ by virtue of (3.6), it follows that (3.8c) holds after adding a suitable multiple of $|D \psi|_{0}$ to $\tau_{0}$. The extended version of $(3.11 \mathrm{a})$ is obvious because $\theta_{\delta} \leq 0$.

In some cases, inequality $(6.1)$ can be proved directly from structure conditions analogous to those in $\S 2$. (Such is the case, for example, in [7].) Here we take advantage of the gradient bounds just proved. First of all, let us set $w=\psi-u, \tilde{\varphi}(x)=\varphi(x, u), \bar{A}(x, z, p)=-A(x, \psi-z, D \psi-p)$, and $\bar{B}(x, z, p)=-B(x, u, D u)+\mu \theta_{\delta}(-w)$. By suitable extension of $\bar{A}$, we can find nonnegative constants $\tilde{a}_{1}, \tilde{b}_{1}, \tilde{c}_{0}$ such that

$$
p \cdot \bar{A}(x, z, p) \geq|p|^{2}-\tilde{a}_{1}, \quad w \bar{B} \leq \tilde{b}_{1}, \quad w \varphi \leq \tilde{c}_{0}
$$

wherever $w>2 \delta$. (Of course $\tilde{a}_{1}, \tilde{b}_{1}, \tilde{c}_{0}$ will depend on all the structure used to obtain our gradient bound.) We now apply Lemma 2.1 with $m=2, M=$ $2 \delta, q=2 n, a_{1}=\tilde{a}_{1} \delta^{-2}, b_{1}=\tilde{b}_{1} \delta^{-2}, c_{0}=\tilde{c}_{0} \delta^{-1}$, and $b_{0}=0$ to infer that

$$
\sup _{\Omega} w \leq c\left(\tilde{a}_{1}, \tilde{b}_{1}, \tilde{c}_{0}, n, q, \Omega\right)\left[\left(\delta^{-n} \int_{\{w>2 \delta\}} w^{2 n} d x\right)^{1 / 2 n}+\delta\right] \text {. }
$$

In fact, for $\mu \geq \tilde{b}_{1} / \delta$ we have

$$
w \bar{B} \leq \frac{1}{2} \mu w
$$

so Lemma 2.2 with $m=2, m_{0}=1, M_{0}=2 \delta, q=2 n, a_{1}=\tilde{a}_{1} / \delta, b_{0}=$ $0, b_{1}=\mu / 2$, and $c_{0}=\tilde{c}_{0}$ gives

$$
\int_{\{w>2 \delta\}} w^{2 n} d x \leq c(n, \Omega) \delta^{2 n}
$$

provided $\mu$ is sufficiently large (depending also on $\delta$ ). The combination of (6.2) and (6.3) with the inequality $0<\delta<1$ gives (6.1). 
When $\Gamma$ is empty and $\partial \Omega \in C^{2, \alpha}$ for some $\alpha \in(0,1)$, solutions of the penalized problem exist by virtue of $[21, \S 7]$ under the additional hypotheses listed there. Standard approximation arguments (see §7) allow us to relax the regularity hypotheses on $\partial \Omega$ and the functions $A, B$, and $\varphi$ to those needed for our estimates. In particular, we can allow the singular set $\Gamma$ to be nonempty.

As in [7, 6, 23], etc., adding the hypotheses

$$
\begin{aligned}
& A(x, \psi, D \psi) \cdot \gamma+\varphi(x, \psi) \leq 0 \text { on } \partial \Omega, \\
& \varphi \in C^{1, \eta}, B, A_{z}, A_{x} \text { in } C^{\eta}, a^{i j} \in C^{0}, \partial \Omega \in C^{2, \eta}
\end{aligned}
$$

for some $\eta \in(0,1]$, gives second derivative bounds up to $\partial \Omega$.

\section{SOME REMARKS ON EXISTENCE}

In deriving our gradient bound, we had to assume that the solution was Lipschitz, that is, the gradient was bounded. In the case of a smooth boundary, this assumption is not serious because strong existence results are known; however, for a nonsmooth domain, this assumption is important.

In a convex domain (in two dimensions) near a corner, solutions of conormal derivative problems generally have bounded (and even continuous) gradients while their gradients are generally unbounded near a nonconvex corner. For example, let $\Omega$ be the set, in polar coordinates, given by $0<r<1,0<\theta<\frac{3 \pi}{2}$ and write

$$
\Sigma_{1}=\left\{r=1,0<\theta<\frac{3 \pi}{2}\right\}, \quad \Sigma_{2}=\left\{0<r<1, \theta=0, \theta=\frac{3 \pi}{2}\right\} .
$$

Then $u=r^{2 / 3} \cos \frac{2}{3} \theta$ is the unique solution up to an additive constant of

$$
\Delta u=0 \quad \text { in } \Omega, \quad \frac{\partial u}{\partial \gamma}=-\frac{2}{3} \cos \frac{2}{3} \theta \quad \text { on } \Sigma_{1}, \quad \frac{\partial u}{\partial \gamma}=0 \quad \text { on } \Sigma_{2} .
$$

At the nonconvex corner $\{r=0\}$, the gradient is unbounded, while at the convex corners $\left\{r=1, \theta=0\right.$ or $\left.\frac{3 \pi}{2}\right\}, u$ has Hölder continuous gradient; in fact, $u$ is $C^{\infty}$ everywhere except at the origin. The estimates of $\S 3$ do not distinguish between the convex and nonconvex corners, but they can only be applied when the solution is known to be Lipschitz.

Two approaches are possible in proving that a solution has bounded gradient. First, the approach used by the author in, e.g., [21] can be applied provided the only singularities of $\partial \Omega$ are edges and the interior angle between the hypersurfaces meeting at each edge is less than $\pi$. The only change in the proof is that the linear theory of [22], which is valid in such domains, replaces the classical Schauder theory. Hence, with this additional assumption on the domain, the hypotheses of $\S \S 2$ and 3 and the conditions

$$
\begin{gathered}
A \in C^{1+\alpha}\left(\bar{\Omega} \times \mathbb{R} \times \mathbb{R}^{n}\right), \quad B \in C^{\alpha}\left(\bar{\Omega} \times \mathbb{R} \times \mathbb{R}^{n}\right), \\
A_{p} \in C^{1+\delta}\left(\bar{\Omega} \times \mathbb{R} \times \mathbb{R}^{n}\right), \\
B_{z} \text { and } B_{p} \text { are in } C^{\delta}\left(\bar{\Omega} \times \mathbb{R} \times \mathbb{R}^{n}\right), \\
\varphi \in C^{1+\alpha}(\partial \Omega \times \mathbb{R}),
\end{gathered}
$$




$$
\begin{gathered}
\left(a^{i j}\right)>0 \quad \text { on } \Omega \times \mathbb{R} \times \mathbb{R}^{n}, \\
A_{z}=0, B_{z}<0 \quad \text { on } \Omega \times \mathbb{R} \times \mathbb{R}^{n}, \\
\varphi_{z} \leq 0 \quad \text { on } \partial \Omega \times \mathbb{R}
\end{gathered}
$$

for some positive $\alpha, \delta$ imply that $(0.1)$ has a unique $C^{1+\varepsilon}(\bar{\Omega})$ solution for suitable $\varepsilon>0$. The local results in $\S 3$ are useful in this case primarily for showing that the bound on the gradient depends only on local data. As pointed out in [21], conditions (7.1), (7.2), and (7.3) can be modified somewhat. Thus, if the only singularities of $\partial \Omega$ are edges and if conditions $(2.1)^{\prime},(3.1)-(3.12)$, (7.1), (7.2), and (7.3) hold, then (0.1) has a unique solution, which has bounded gradient. (Of course if $m=1$ in $(2.1)^{\prime}$, we should replace $(2.1 \mathrm{c})^{\prime}$ by $(2.1 \mathrm{c})^{\prime \prime}$.)

An alternative approach is needed to consider domains with other types of singularities. The approximation scheme sketched by Korevaar in $[9, \S 4]$ is appropriate for this situation.

From the appendix there is a function $\rho \in C^{0,1}(\bar{\Omega}) \cap C^{\infty}(\Omega)$ which is equivalent to $d$. Since $\Omega$ satisfies an exterior sphere condition and an interior cone condition, there are positive constants $\varepsilon_{0}$ and $\rho_{0}$ such that $|D \rho|>\rho_{0}$ for $0<\rho<\varepsilon_{0}$ and, if $\Omega_{\varepsilon}=\{x \in \Omega: \rho(x)>\varepsilon\}$ with inner normal $\gamma_{\varepsilon}$, we have $\partial \Omega_{\varepsilon} \in C^{\infty}, \bar{\gamma} \cdot \gamma_{\varepsilon} \geq C(\Omega) \gamma_{0}$, and

$$
D_{i} \gamma_{\varepsilon, j} \zeta^{i} \zeta^{j} \leq \frac{20}{\rho_{0} R}|\zeta|^{2}
$$

for $\zeta \cdot \gamma_{\varepsilon}=0$ and $0<\varepsilon<\varepsilon_{0}$. Hence if conditions $(2.1)^{\prime},(3.1)-(3.12)$, (7.1a, b, c), (7.2), (7.3) and

$$
\bar{\varphi} \in C^{1+\alpha}(\partial \Omega \times \mathbb{R})
$$

hold for $(0.1)$, the boundary value problem

$$
\begin{aligned}
\operatorname{div} A\left(x, u_{\varepsilon}, D u_{\varepsilon}\right)+B\left(x, u_{\varepsilon}, D u_{\varepsilon}\right)=0 & \text { in } \Omega_{\varepsilon}, \\
A\left(x, u_{\varepsilon}, D u_{\varepsilon}\right) \cdot \gamma_{\varepsilon}+\bar{\gamma} \cdot \gamma_{\varepsilon} \bar{\varphi}=0 & \text { on } \partial \Omega_{\varepsilon}
\end{aligned}
$$

has a unique solution $u_{\varepsilon} \in C^{2+\alpha}\left(\bar{\Omega}_{\varepsilon}\right)$. From $\S 3,\left|D u_{\varepsilon}\right|$ is bounded uniformly as $\varepsilon \rightarrow 0$, so $u_{\varepsilon}$ converges uniformly to the unique solution of $(0.1)$. Standard regularity shows that $u \in C^{2+\alpha}(\Omega)$, it follows from [19] that $u \in C^{1+\theta}(\bar{\Omega} \backslash \Gamma)$ for $\theta \in(0,1)$, and [20] implies that $D u$ is Hölder continuous near interior points of edges of $\partial \Omega$ as well.

\section{APPENDIX. REMARKS ON REGULARIZED DISTANCE}

In this appendix, we construct a regularized distance for $\Omega$, i.e., a function $\rho \in C^{0,1}\left(\mathbb{R}^{n}\right) \cap C^{2}\left(\mathbb{R}^{n} \backslash \partial \Omega\right)$ which is equivalent to the signed distance function $d$ defined by

$$
d(x)= \begin{cases}\operatorname{dist}(x, \partial \Omega) & \text { if } x \in \Omega, \\ -\operatorname{dist}(x, \partial \Omega) & \text { if } x \notin \Omega .\end{cases}
$$

To prove our results, we recall the construction of regularized distance from [14, Lemma 1.1] with a slight modification to take account of a more detailed 
structure. Let $\varphi$ be a nonnegative $C^{2}\left(\mathbb{R}^{n}\right)$ function supported in the unit ball with $\int \varphi(z) d z=1$. If $g$ is a Lipschitz function such that $g / d$ and $d / g$ are uniformly bounded and positive in $\mathbb{R}^{n} \backslash \partial \Omega$, and $L$ and $M$ are positive constants such that $|g(x)-g(y)| \leq M|x-y|$ for all $x$ and $y$ in $\mathbb{R}^{n}$ and $L>M$, we define

$$
G(x, \tau)=\int g(x-\tau z / L) \varphi(z) d z
$$

for $(x, \tau) \in \mathbb{R}^{n+1}$. A regularized distance $\rho$ is then given by the equation $\rho(x)=G(x, \rho(x))$. To see that this equation defines $\rho$ uniquely, we note from formulas (1.3) and (1.4) in [14] that the partial derivatives $G_{x}$ and $G_{\tau}$ satisfy the inequalities

$$
\left|G_{x}(x, \tau)\right| \leq M, \quad\left|G_{\tau}(x, \tau)\right| \leq M / L<1 .
$$

We also note that

$$
D \rho(x)=\frac{G_{x}(x, \rho(x))}{1-G_{\tau}(x, \rho(x))} .
$$

We now present two definitions. If $x_{0} \in \partial \Omega$ and if there are positive constants $\delta, A$, an orthonormal coordinate system $Y=\left(y^{\prime}, y^{n}\right)=\left(y^{1}, \ldots, y^{n}\right)$, and a function $f: \mathbb{R}^{n-1} \rightarrow \mathbb{R}$ with

$$
\left|f\left(y_{1}^{\prime}\right)-f\left(y_{2}^{\prime}\right)\right| \leq A\left|y_{1}^{\prime}-y_{2}^{\prime}\right| \text { for }\left|y_{1}^{\prime}\right|,\left|y_{2}^{\prime}\right|<4 \delta
$$

such that

$$
\{y \in \Omega:|y|<4 \delta\}=\left\{y \in \mathbb{R}^{n}: y^{n}>f\left(y^{\prime}\right),|y|<4 \delta\right\},
$$

we call $f$ a local representation of $\partial \Omega$ near $x_{0}$. If $\partial \Omega$ has a local representation near each point $x_{0}$ of $\partial \Omega$ with constants $\delta$ and $A$ independent of $x_{0}$, we say $\Omega$ is Lipschitz. (More commonly, such a domain is called uniformly Lipschitz.) Note that a domain is Lipschitz if and only if it satisfies uniform interior and exterior cone conditions.

Lemma A.1. Any Lipschitz domain $\Omega$ has a proper regularized distance $\rho$. Moreover, if $0<|d(x)|<\delta$, then $\frac{3}{2}|D \rho(x)| \geq\left(1+A^{2}\right)^{-1 / 2}$.

Proof. Let $x \in \Omega$ with $d(x)<\delta$ and choose $x_{0} \in \partial \Omega$ so that $\left|x-x_{0}\right|=d(x)$. Working in the $Y$ coordinate system corresponding to $x_{0}$, we first show that if $\left|y_{1}\right|,\left|y_{2}\right|<2 \delta$ with $y_{1}^{\prime}=y_{2}^{\prime}$ and $y_{1}^{n}>y_{2}^{n} \geq f\left(y_{1}^{\prime}\right)$, then

$$
d\left(y_{1}\right)-d\left(y_{2}\right) \geq \frac{y_{1}^{n}-y_{2}^{n}}{\left(1+A^{2}\right)^{1 / 2}} .
$$

To prove (A.4), let $y_{0} \in \partial \Omega$ such that $d\left(y_{1}\right)=\left|y_{1}-y_{0}\right|$, note that $\left|y_{0}^{\prime}\right|<4 \delta$, and let $K$ be the exterior cone to $\Omega$ with vertex $y_{0}$, vertex angle $2 \operatorname{arccot} A$, and axis parallel to the $y^{n}$-axis. Then

$$
d\left(y_{1}\right)=\operatorname{dist}\left(y_{1}, K\right), \quad d\left(y_{2}\right) \leq \operatorname{dist}\left(y_{2}, K\right) .
$$

Now write $\mathscr{L}$ for the line through $y_{2}$ and parallel to the line segment $y_{0} y_{1}$, $z_{0}$ for the point of intersection of $\mathscr{L}$ with the axis of $K$ and $z_{1}$ for the point of intersection of $\mathscr{L}$ with $K$, which is closer to $y_{2}$. It follows that the cotangent of the angle $z_{0} y_{0} z_{1}$ is $A$, so $\left|z_{0}-z_{1}\right| \geq\left(1+A^{2}\right)^{-1 / 2}\left|y_{0}-z_{0}\right|$. Since 
$\left|y_{0}-z_{0}\right|=y_{1}^{n}-y_{2}^{n}, \operatorname{dist}\left(y_{2}, K\right) \leq\left|y_{2}-z_{1}\right|$, and $\operatorname{dist}\left(y_{1}, K\right)=\left|y_{2}-z_{0}\right|=$ $\left|y_{2}-z_{1}\right|+\left|z_{1}-z_{0}\right|$, we also have

$$
\operatorname{dist}\left(y_{1}, K\right)-\operatorname{dist}\left(y_{2}, K\right) \geq \frac{y_{1}^{n}-y_{2}^{n}}{\left(1+A^{2}\right)^{1 / 2}} .
$$

The combination of (A.5) and (A.6) gives (A.4).

Now take $g=d$ and $M=1$ in (A.1). If $X^{\prime}=x^{\prime}$ and $f\left(x^{\prime}\right)<X^{n}<x^{n}$, then

$$
G(x, \tau)-G(X, \tau)=\int\left[d\left(x-\frac{\tau z}{L}\right)-d\left(X-\frac{\tau z}{L}\right)\right] \varphi(z) d z \geq \frac{x^{n}-X^{n}}{\left(1+A^{2}\right)^{1 / 2}}
$$

provided $0<\tau<L \min \{d(X), d(x)\}$. Hence if we divide this inequality by $x^{n}-X^{n}$ and let $X^{n}$ approach $x^{n}$, we find that the derivative $G_{n}=\partial G / \partial x^{n}$ satisfies the estimate $G_{n}(x, \tau) \geq\left(1+A^{2}\right)^{-1 / 2}$ for $0<\tau<L d(x)$, and by continuity of $G_{n}$, also for $\tau=L d(x)$. In particular, we can use $\tau=\rho(x)$ to infer that

$$
G_{n}(x, \rho(x)) \geq\left(1+A^{2}\right)^{-1 / 2} .
$$

From (A.2), (A.3), and (A.7), we have $D_{n} \rho(x) \geq \frac{L}{(L+M)\left(1+A^{2}\right)^{1 / 2}}$ for $0<d(x)<$ $\delta$, and a similar argument works if $-\delta<d(x)<0$. Choosing $L=2$ gives the estimate on $|D \rho|$.

Note that by choosing $L$ sufficiently large, we can make $|D \rho| \geq \alpha\left(1+A^{2}\right)^{-1 / 2}$ for any $\alpha \in(1 / 2,1)$.

The method of $[14, \S 2]$ gives an alternative proof of Lemma 1 with different constants by constructing local regularized distances and then piecing them together via a partition of unity.

When the exterior cone condition is strengthened to an exterior sphere condition, the regularized distance just constructed satisfies a one-sided second derivative condition. The proof of this fact uses the following consequence of [14, (1.10)]: Let $x \in \mathbb{R}^{n} \backslash \partial \Omega$ and $\xi \in \mathbb{R}$, set $\Xi=(\xi, \xi \cdot D \rho(x))$, and use subscripts $i=1, \ldots, n+1$ on $G$ to denote partial derivatives with respect to $x^{i}$ if $i \leq n$ and with respect to $\tau$ if $i=n+1$. Then

$$
D_{i j} \rho(x) \xi^{i} \xi^{j}=\frac{G_{i j}(x, \rho(x)) \Xi^{i} \Xi^{j}}{1-G_{n+1}(x, \rho(x))} .
$$

(Here and below we follow the summation convention that repeated indices are to be summed over the appropriate range. For example, in (A.8) the indices $i$ and $j$ are summed from 1 to $n$ on the left-hand side but from 1 to $n+1$ on the right.)

Theorem A.2. If $\Omega$ satisfies a uniform exterior sphere condition with radius $R$, then $\Omega$ has a regularized distance $\rho$ such that

$$
D_{i j} \rho(x) \xi^{i} \xi^{j} \leq \frac{C(n)}{R}|\xi|^{2}
$$

for all $\xi \in \mathbb{R}^{n}$ and all $x \in \mathbb{R}^{n} \backslash \partial \Omega$, and

$$
D_{i j} \rho(x) \xi^{i} \xi^{j} \leq \frac{4}{R}|\xi|^{2}
$$


for all $\xi \in \mathbb{R}^{n}$ and all $x \in \Omega$. If $\Omega$ satisfies a uniform interior cone condition, then $\rho$ is proper.

Proof. We first prove that

$$
d(x+h)+d(x-h)-2 d(x) \leq \frac{|h|^{2}}{R}
$$

for all $x \in \Omega$ and all $h \in \mathbb{R}^{n}$ with $|h|<d(x)$. To prove this inequality, for each $y \in \partial \Omega$, write $z$ for the center of the exterior sphere to $\Omega$ at $y$ with radius $R$, and define $d_{y}$ by $d_{y}(x)=|x-z|-R$. Note that $d \leq d_{y}$ and $d_{y} \in C^{2}\left(\mathbb{R}^{n} \backslash\{z\}\right)$ for any $y \in \partial \Omega$. Therefore

$$
D_{i j} d_{y}(x) h^{i} h^{j}=\frac{|h|^{2}}{|x-z|}-\frac{|(x-z) \cdot h|^{2}}{|x-z|^{3}} \leq \frac{|h|^{2}}{|x-z|} \leq \frac{|h|^{2}}{R} .
$$

For $|h|<d_{y}(x)$, Taylor's Theorem shows that $d_{y}(x+h)+d_{y}(x-h)-2 d_{y}(x) \leq$ $\frac{|h|^{2}}{R}$. If we choose $y$ so that $d(x)=|x-y|$, then $d(x)=d_{y}(x)$. Since $d_{y} \leq d$, (A.11) follows immediately.

From (A.11) and (A.1) with $g=d$ and $\varphi$ even, so that $\int z \varphi(z) d z=0$, we see that

$$
G(x+h, \tau+k)+G(x-h, \tau-k)-2 G(x, \tau) \leq \frac{|h|^{2}+k^{2} / L^{2}}{R}
$$

for $0<\tau<L d(x) / 2$ and $|h|+|k| / L \leq d(x) / 2$. Dividing by $|h|^{2}+k^{2} / L^{2}$, letting this denominator tend to zero, and choosing $\tau=\rho(x)$ gives

$$
G_{i j}(x, \rho(x)) \Xi^{i} \Xi^{j} \leq \frac{\left|\Xi^{\prime}\right|^{2}+\left(\Xi^{n+1} / L\right)^{2}}{R}
$$

for any $\Xi=\left(\Xi^{\prime}, \Xi^{n+1}\right) \in \mathbb{R}^{n+1}$. It now follows from (A.2), (A.3), and (A.8) that

for $L=2, M=1$.

$$
D_{i j} \rho(x) \xi^{i} \xi^{j} \leq \frac{L\left(|\xi|^{2}+|\xi \cdot D \rho(x)|^{2}\right) / L^{2}}{(L-M) R} \leq \frac{4|\xi|^{2}}{R}
$$

When $-R / 2<d(x)<0$, we have $|x-z| \geq R / 2$ and hence the preceding argument shows that $D_{i j} \rho(x) \xi^{i} \xi^{j} \leq \frac{8}{R}|\xi|^{2}$.

Finally, it follows from the proof of [14, Theorem 1.3] that $\left|D^{2} \rho\right| \leq C^{\prime}(n) / d$, and therefore $d(x)<-R / 2$ implies that (A.9) holds with $C$ replaced by $C^{\prime}$. Hence (A.9) holds in all cases if $C=\max \left\{8, C^{\prime}\right\}$.

Lemma A.1 implies that $\rho$ is proper in case of an interior cone condition.

By choosing $L$ sufficiently large, we can replace 4 in inequality (A.10) by any constant greater than 1 .

We now show how to infer $(3.12)^{\prime}$ from (3.12). Suppose that $\Omega$ is a Lipschitz domain satisfying an exterior sphere condition with radius $R$ and let $\rho$ be the regularized distance constructed in Lemma A.1. With $\delta$ as in the definition of Lipschitz domain and $\varepsilon \in(0, \delta)$, write $\gamma$ for the normal to the surface $\Sigma=\{x: \rho(x)=\varepsilon\}$ and let $\xi$ be a vector field tangent to $\Sigma$. Since $\gamma=D \rho /|D \rho|$ on $\Sigma$, we see from (A.3), (A.7), (A.8), and (A.12) that

$$
\begin{aligned}
D_{i} \gamma_{j} \xi^{i} \xi^{j} & =\frac{1}{|D \rho|} D_{i j} \rho \xi^{i} \xi^{j}=\frac{1}{\left|G_{x}(x, \rho(x))\right|} G_{i j}(x, \rho(x)) \Xi^{i} \Xi^{j} \\
& \leq\left(1+A^{2}\right)^{1 / 2} \frac{\left|\Xi^{\prime}\right|^{2}}{R}=\left(1+A^{2}\right)^{1 / 2} \frac{|\xi|^{2}}{R} \quad \text { on } \Sigma .
\end{aligned}
$$


In a sufficiently small neighborhood of $x_{0} \in \partial \Omega \backslash \Gamma$, we can make $A$ arbitrarily close to zero by choosing $\delta$ small enough. Hence for any constant $C>1$, we have $D_{i} \gamma_{j} \zeta^{i} \zeta^{j} \leq C \frac{|\zeta|^{2}}{R}$, and sending $C$ to 1 proves $(3.12)^{\prime}$.

\section{REFERENCES}

1. R. Finn, Equilibrium capillary surfaces, Springer-Verlag, Berlin and New York, 1986.

2. M. Giaquinta and E. Giusti, Global $C^{1, \alpha}$-regularity for second order elliptic equations in divergence form, J. Reine Angew. Math. 351 (1984), 55-65.

3. C. Gerhardt, Global regularity of solutions to the capillarity problem, Ann. Scuola Norm Sup. Pisa Cl. Sci. (3) 4 (1976), 157-176.

4. D. Gilbarg and L. Hormander, Intermediate Schauder estimates, Arch. Rational Mech. Anal 74 (1980), 297-318.

5. D. Gilbarg and N. S. Trudinger, Elliptic partial differential equations of second order, 2nd Ed., Springer-Verlag, Berlin and New York, 1983.

6. B. Huisken, Second order boundary regularity for quasilinear variational inequalities, Manuscripta Math. 63 (1989), 333-342.

7. G. Huisken, Capillarity surfaces over obstacles, Pacific J. Math. 117 (1985), 121-141.

8. L. I. Kamynin and B. N. Khimchenko, The principle of the maximum and boundary Lipschitz bounds for the solution of a second order elliptic-parabolic equation, Siberian Math. J. 15 (1974), 242-260.

9. N. J. Korevaar, Maximum principle gradient estimates for the capillary problem, Comm. Partial Differential Equations 13 (1988), 1-31.

10. O. A. Ladyzhenskaya and N. N. Ural'tseva, Linear and quasilinear elliptic equations, Izdat. "Nauka", Moscow, 1964; English transl., Academic Press, New York, 1968.

11. G. M. Lieberman, The quasilinear Dirichlet problem with decreased regularity at the boundary, Comm. Partial Differential Equations 6 (1981), 437-467.

12. _ The conormal derivative problem for elliptic equations of variational type, J. Differential Equations 49 (1983), 218-257.

13. _ Interior gradient bounds for non-uniformly parabolic equations, Indiana Univ. Math. J. 32 (1983), 579-601.

14. __ Regularized distance and its applications, Pacific J. Math. 117 (1985), 329-353.

15. __ Quasilinear elliptic equations with nonlinear boundary conditions, Proc. Sympos. Pure Math., vol. 45, part 2, Amer. Math. Soc., Providence, R.I., 1986, pp. 113-117.

16. Intermediate Schauder theory for oblique derivative problems, Arch. Rational Mech. Anal 93 (1986), 129-134.

17. _ The Dirichlet problem for quasilinear elliptic equations with continuously differentiable boundary data, Comm. Partial Differential Equations 11 (1986), 167-229.

18. __ Local estimates for subsolutions and supersolutions of oblique derivative problems for general second-order elliptic equations, Trans. Amer. Math. Soc. 304 (1987), 343-353.

19. __ Hölder continuity of the gradient of solutions of uniformly parabolic equations with conormal boundary conditions, Ann. Mat. Pura Appl. (4) 148 (1987), 77-99, 397-398.

20. _ Hölder continuity of the gradient at a corner for the capillary problem and related results, Pacific J. Math. 133 (1988), 115-135.

21. The conormal derivative problem for non-uniformly parabolic equations, Indiana Univ. Math. J. 37 (1988), 23-72.

22. __ Oblique derivative problems in Lipschitz domains. II. Discontinuous boundary data, J. Reine Angew. Math. 389 (1988), 1-24.

23. _ Boundary regularity for linear and quasilinear variational inequalities, Proc. Roy. Soc. Edinburgh 112 (1989), 319-326.

24. N. G. Meyers, $A$ theory of capacities for potentials of functions in Lebesgue classes, Math. Scand. 26 (1970), 255-292. 
25. J. H. Michael and L. Simon, Sobolev and mean value inequalities on generalized submanifolds of $R^{n}$, Comm. Pure Appl. Math. 26 (1973), 361-379.

26. J. Serrin, Local behavior of solutions of quasilinear equations, Acta Math. 111 (1964), 247-302.

27. _ Gradient estimates for solutions of nonlinear elliptic and parabolic equations, Contributions to Nonlinear Functional Analysis, Academic Press, 1971, pp. 565-602.

28. L. Simon, Interior gradient bounds for non-uniformly elliptic equations, Indiana Univ. Math. J. 25 (1976), 821-855.

29. N. N. Ural'tseva, Solvability of the capillary problem, Vestnik Leningrad. Univ. 19 (1973), 54-64; 1 (1975), 143-149; English transl., Vestnik Leningrad Univ. Math. 6 (1979), 363$375 ; 8$ (1980), 151-158.

30. __ Estimates for the maxima of the moduli of the gradients for solutions of capillarity problems, Zap. Nauchn. Sem. Leningrad. Otdel. Mat. Inst. Steklov (LOMI) 115 (1982), 274-284; English transl., J. Soviet Math. 28 (1985), 806-813.

31. G. M. Lieberman, The natural generalization of Ladyzhenskaya and Ural'tseva for elliptic equations, Comm. Partial Differential Equations 16 (1991), 311-361.

Department of Mathematics, Iowa State University, Ames, Iowa 50011 